Revista de Matemática: Teoría y Aplicaciones 2018 25(1) : 79-113

CIMPA - UCR ISSN: 1409-2433 (PRINT), 2215-3373 (ONLINE)

\title{
UN MODELO DE CRECIMIENTO POBLACIONAL DE ÆDES ÆGYPTI CON CAPACIDAD DE CARGA LOGÍSTICA
}

\author{
A POPULATION GROWTH MODEL OF ÆDES \\ ÆGYPTI WITH LOGISTIC CARRYING CAPACITY
}

Julián Alejandro Olarte García*

AnÍBAL MuÑOZ LOAIZA ${ }^{\dagger}$

Received: 17/Feb/2017; Revised: 10/Aug/2017;

Accepted: $18 /$ Oct/2017

Revista de Matemática: Teoría y Aplicaciones is licensed under a Creative Commons Reconocimiento-NoComercial-Compartirigual 4.0 International License.

Creado a partir de la obra en http://www.revistas.ucr.ac.cr/index.php/matematica

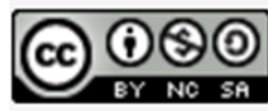

* Grupo de Modelación Matemática en Epidemiología (GMME), Facultad de Educación, Universidad del Quindío, Armenia, Colombia. E-Mail: jaolarteg@uqvirtual.edu.co

${ }^{\dagger}$ Misma dirección que/Same address as: J.A. Olarte.

E-Mail: anibalml@hotmail.com 


\title{
Resumen
}

En esta investigación se plantea y analiza un modelo matemático mediante un sistema de ecuaciones diferenciales ordinarias no lineales que representa la bioecología de los mosquitos Aedes aegypti, por cuanto involucra su ciclo de vida y sus sitios reproductivos principales. Se hallan las soluciones constantes del sistema en términos del umbral de crecimiento poblacional, haciéndose el análisis de estabilidad a cada una. Se realiza un análisis de sensibilidad local del umbral de crecimiento y del equilibrio de coexistencia en términos de los parámetros demográficos contemplados en la dinámica. Con datos calculados e hipotéticos, se presentan resultados numéricos de las soluciones del sistema, los cuales se obtienen con ayuda del software matemático Maple.

Palabras clave: Aedes aegypti; criadero; modelo logístico; umbral de crecimiento; estabilidad local y global; análisis de sensiblidad..

\begin{abstract}
In this research, a mathematical model is proposed and analyzed by means of a system of nonlinear ordinary differential equations that represents the bioecology of the Aedes aegypti mosquitoes, since it involves their life cycle and their main breeding sites. The constant solutions of the system are found in terms of the population growth threshold, with the stability analysis being performed to each one. A local sensitivity analysis of the growth threshold and the coexistence equilibrium is performed in terms of the demographic parameters contemplated in the dynamics. With calculated and hypothetical data, numerical results of the system solutions are presented, which are obtained with the help of the mathematical software Maple.
\end{abstract}

Keywords: Aedes aegypti; breeding site; logistic model; growth threshold; local and global stability; sensitivity analysis.

Mathematics Subject Classification: 92B05, 92C60.

\section{Introducción}

Aedes aegypti es una especie tropical y subtropical del subgénero Stegomyia y de la familia Culicidae, al parecer nativa de África, ampliamente distribuida por el mundo. Sus hábitos son netamente antropófilos y domésticos, con radicación de criaderos en la vivienda humana o en sus inmediaciones [23]. Es el vector responsable de la transmisión de enfermedades víricas como el dengue, la fiebre amarilla, la fiebre chikungunya y el virus Zika a los seres humanos [29].

Se distribuye en forma permanente entre las latitudes $35^{\circ} \mathrm{N}$ y $35^{\circ} \mathrm{S}$, lo que corresponde, aproximadamente, a una isoterma de invierno de $10^{\circ} \mathrm{C}$, pero puede 
extenderse hasta los $45^{\circ} \mathrm{N}$ y hasta los $40^{\circ} \mathrm{S}$, encontrándose en una altitud promedio por debajo de los $1.800 \mathrm{msnm}$, aunque se ha registrado en alturas de alrededor de los 2.200 msnm [23, 11]. Sus condiciones mínimas de sobrevivencia y su resistencia a diferentes eventos adversos, como la desecación y la inanición, lo hace un mosquito de presencia muy común y continua, así como de elevadas densidades poblacionales durante las épocas lluviosas con temperatura y humedad estables [33].

Los mosquitos se encuentran en pleno desarrollo morfológico en recipientes artificiales que acumulan agua, lluvia o vegetación muy próximos a viviendas $\mathrm{y}$, a menudo, en espacios interiores que son menos susceptibles a variaciones climáticas, lo que aumenta la longevidad de los mosquitos [28]. Dicha situación vuelve vulnerable a los seres humanos, especialmente durante los periodos en que se intensifican las picaduras, el principio de la mañana y el atardecer, antes de que oscurezca [27]. Como consecuencia, los mosquitos adquieren agentes patógenos al succionar la sangre de un vertebrado infectado, y los pueden inocular a un nuevo portador al ingerir su sangre, propagando rápidamente el virus en las comunidades donde radican o de una comunidad a otra [28].

Su ciclo de vida varía entre 7 y 14 días aproximadamente, e incluye la fase acuática, conformada por los huevos (ovipuestos al nivel del agua, no siempre agua limpia y que pueden resistir la sequedad varios meses hasta un año), las larvas y las pupas (que pueden nadar y se alimentan de microorganismos y material orgánico disuelto en el agua), y la fase aérea, conformada por los mosquitos adultos (que pueden volar, los machos son nectívoros y las hembras son hematófagas) $[5,13]$. En su lucha antivectorial, las entidades de salud pública recurren a varios tipos de controles, en esencia tres, los demás son combinaciones de ellos [26]:

- La gestión ambiental es el procedimiento aplicado para evitar o reducir al mínimo la propagación vectorial y el contacto entre las personas y los vectores patógenos, efectuando modificaciones en el ambiente para eliminar permanentemente (modificación del ambiente) o de forma temporal (manipulación del ambiente) el hábitat de los transmisores [30].

- El control biológico es la utilización de organismos patógenos, parásitos, parasitoides o depredadores, enemigos naturales de las especies biológicas plaga o vectores de enfermedades, para mantener a sus poblaciones a niveles inferiores de lo que estarían en su ausencia. Sólo ciertas especies 
de peces larvívoros y copépodos depredadores (Copepoda Cyclopoidea) - pequeños crustáceos de agua dulce- han demostrado ser efectivas contra los vectores Aedes en el contexto de operaciones en hábitats de recipientes específicos, y aún así, muy pocas veces han sido efectivos a gran escala [30].

- El control químico es el procedimiento aplicado contra los vectores, en sus estadios larvarios o inmaduros y de imagos o adultos, utilizando sustancias tóxicas con efecto insecticida, larvicida o adulticida. Debido a que A. aegypti frecuentemente deposita los huevos en recipientes para almacenar agua, los larvicidas deben tener una baja toxicidad para otras especies y no deben cambiar significativamente el sabor, olor ni color del agua [30].

Claramente a través del primer método, se pueden destruir los criaderos favorables para la oviposición y que permiten el desarrollo de las etapas acuáticas. Este método depende de la participación comunitaria para su eficacia [37]. Muchas investigaciones han identificado y caracterizado los criaderos que más aportan a la producción de formas inmaduras de culícidos.

En [24] para la ciudad de Posadas, Argentina, han detectado entre los contenedores más comunes y con mayor presencia de larvas los neumáticos usados, por la elevada productividad de estadios preadultos de dípteros hematófagos y protección que ofrecen a los huevos. En [1] determinaron la productividad de A. aegypti en viviendas y espacios públicos de Girardot (Colombia), en época de lluvias y de sequía. La productividad de pupas en las albercas y tanques bajos de las viviendas fue elevada tanto en la época de sequía como en la de lluvias, en tanto que los espacios públicos contribuyeron con más sitios de cría durante la época de lluvias. En [19] se identificó la presencia de A. aegypti en los seis cantones de la provincia de Limón, Costa Rica. Los sitios de cría más frecuentes fueron los neumáticos, los tanques y los baldes.

En un estudio realizado en la ciudad de Corrientes, Argentina, se seleccionó como lugares de muestreo para la cría de A. aegypti, domicilios, baldíos, cementerios, gomerías, plazas, mercados, chacaritas y gasolineras. Teniendo en cuenta la relación: recipientes encontrados/recipientes positivos, los neumáticos representaron el criadero preferido para inmaduros de culícidos, siguiendo en importancia frascos de vidrio de tamaños variables, recipientes de plástico de hasta un litro y recipientes de plástico de 1 a 10 litros [42]. En un estudio en Mérida (México) se determinó la importancia de los criaderos de A. aegypti; los tipos de recipientes más productivos, en promedio $90 \%$ de las pupas durante todo el año, resultaron ser las cubetas (54.2\%), los diversos objetos chicos $(18.5 \%)$, las macetas (11.5\%) y los diversos objetos grandes (6.4\%), de los 
cuales $77 \%$ era de utilidad para los moradores, mientras que el restante $23 \%$ era desechable [3].

En [20] A. aegypti mostró su mayor preferencia por reproducirse durante todo el periodo 2003-2010 en el que se realizaron colectas larvales en la provincia Pinar del Río, Cuba, en los tanques bajos con el $56.3 \%$ del total acumulado. En [36] se identificaron criaderos de A. aegypti en dos ciudades de la provincia del Chaco (Noreste de Argentina). Los neumáticos y cajas de baterías para autos fueron los recipientes que más aportaron a la producción de formas inmaduras de los culícidos colectados. En [43] se documentaron doce especies de mosquitos en Mérida, siendo A. aegypti la más abundante, y las cubetas como los criaderos más importantes en frecuencia y abundancia de mosquitos. Coherente con los estudios citados, los neumáticos, tanques y baldes son los recipientes más comunes e importantes en términos de densidad y persistencia en la producción de formas inmaduras.

En lo concerniente al modelado matemático del ciclo biológico del mosquito y sus sitios de reproducción, existen trabajos: primero, que evalúan la incidencia del dengue que varía con la temperatura, presentando en primer lugar un modelo compartimental que abarca tres etapas del ciclo de vida del mosquito: larva, pupa y adulto, donde la tasa de producción de larvas efectiva fue dada por una función logística, en que intervienen la capacidad disponible de los recipientes para recibir las larvas de los huevos eclosionados y la capacidad de carga total de los recipientes [40]; segundo, que describen la dinámica de la enfermedad del dengue dentro de las poblaciones preadulta y adulta mediante el desarrollo de un modelo de compartimentos teniendo en cuenta los controles químicos y control mecánico aplicado sobre los mosquitos. Con respecto al control mecánico, se basa en eliminar una fracción de mosquitos inmaduros de cada tipo de recipiente, con una capacidad máxima de almacenamiento [41]; tercero, que estudian la dinámica de crecimiento poblacional del A. Aegypti con base en los modelos tipo presa-depredador y considerando control químico y biológico del mosquito, el cual es considerado como la presa. El proceso integra el ciclo de vida del A. aegypti, el estadio del mosquito maduro y un estadio inmaduro (huevo, larva o pupa). Consideran crecimiento logístico del depredador, capacidad de carga de los criaderos [18].

Hay que resaltar, como alternativa a la capacidad de carga constante, que diversos estudios han utilizado una capacidad de carga tiempo-dependiente para diversas aplicaciones que ha variado sinusoidal, exponencial y linealmente. Coleman et al. (1979) [8] y Rogovchenko \& Rogovchenko (2009) [34] utilizaron una capacidad de carga oscilante para describir los ambientes estacionales; Ikeda \& Yokoi (1980) [15] modelaron el enriquecimiento de un lago por un nutriente 
y Safuan et al. (2011) [35] describieron el microambiente cambiante bajo una oclusión en la piel humana sana, utilizándose en ambos estudios una capacidad de carga con saturación; Meyer (1994) [21] y Meyer \& Ausubel (1999) [22] introdujeron una capacidad de carga que varía logísticamente para modelar el desarrollo tecnológico de una población, con forma similar a la que implementaron Ebert \& Weisser (1997) [10] para el modelado del tamaño corporal de un huésped infectado por parásitos.

\section{El modelo}

Se formula un modelo teórico con base en ecuaciones diferenciales ordinarias no lineales, que describe la dinámica poblacional de A. aegypti y sus criaderos. Las variables contempladas en el modelo son: $x$ : número promedio de individuos de la población de mosquitos hembras maduros al tiempo $t, y$ : número promedio de individuos de la población de mosquitos hembras inmaduros (huevos, larvas y pupas) al tiempo $t$, y $C_{i}(i=1,2,3)$ : número promedio de focos de infestación, baldes $\left(C_{1}\right)$, tanques $\left(C_{2}\right)$ y neumáticos $\left(C_{3}\right)$, al tiempo $t$.

Los parámetros propuestos en el modelo son: $\omega$ : tasa de transición del estadio inmaduro al estadio adulto, $\epsilon$ : tasa de mortalidad de los mosquitos maduros, $\phi$ : tasa de ovoposición de los mosquitos hembras, $f$ : fracción de huevos que dan origen a mosquitos hembras, $\pi$ : tasa de mortalidad de los mosquitos inmaduros (huevos, larvas y pupas), $\alpha_{i}(i=1,2,3)$ : tasa de incremento de baldes, tanques y neumáticos en el medio, respectivamente, $\beta_{i}(i=1,2,3)$ : tasa de eliminación de baldes, tanques y neumáticos en el medio, respectivamente, $\theta_{i}$ $(i=1,2,3)$ : número promedio de huevos, larvas y pupas alojados en baldes, tanques y neumáticos, respectivamente, y $K_{i}(i=1,2,3)$ : capacidad de carga del medio ambiente al $i$-ésimo criadero de mosquitos. La dinámica se representa a través del siguiente flujograma de la Figura 1, donde $\Gamma(y)=1-\frac{y}{K_{y}}$.

Figura 1: Dinámica ecológica.

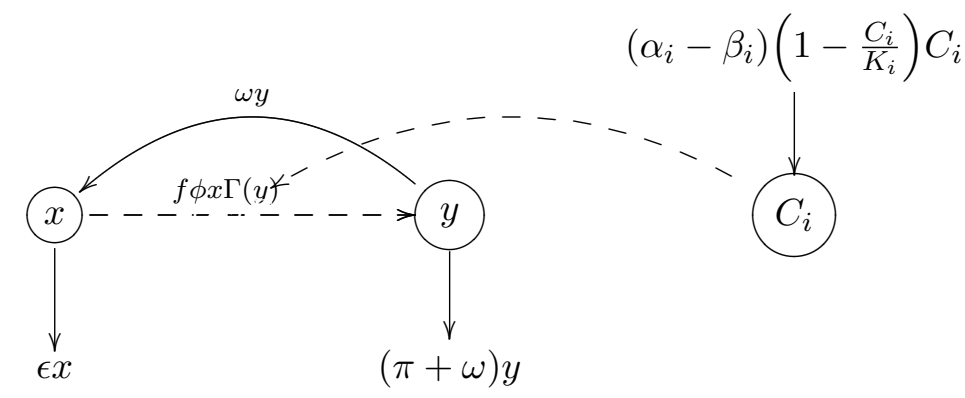

Rev.Mate.Teor.Aplic. (ISSN print: 1409-2433; online: 2215-3373) Vol. 25(1): 79-113, Jan-Jun 2018 
El sistema de ecuaciones diferenciales que la gobierna, escribiendo la variación de cada compartimento como los flujos de entrada menos los flujos de salida, es:

$$
\begin{aligned}
\dot{x} & =\omega y-\epsilon x \equiv \varphi_{1}(x, y) \\
\dot{y} & =f \phi x\left(1-\frac{y}{K_{y}}\right)-(\pi+\omega) y \equiv \varphi_{2}(x, y) \\
\dot{C}_{i} & =\left(\alpha_{i}-\beta_{i}\right)\left(1-\frac{C_{i}}{K_{i}}\right) C_{i} \equiv \varphi_{3}\left(C_{i}\right) \quad(i=1,2,3)
\end{aligned}
$$

donde $\omega, \epsilon, \phi, \pi, \alpha_{i}, \beta_{i}, K_{i}>0,0<f<1$, y condiciones iniciales $x(0)=x_{0}$, $y(0)=y_{0}, C_{i}(0)=C_{i 0}, i=1,2,3$. La región de sentido biológico está definida como:

$\Pi=\left\{\left(y, x, C_{1}, C_{2}, C_{3}\right) \in \mathbb{R}_{+}^{5}: 0 \leq y \leq K_{y}, 0 \leq x \leq \frac{\omega}{\epsilon} K_{y} 0<C_{i} \leq K_{i} ; i=1,2,3\right\}$.

\subsection{Dinámica de la capacidad de carga}

La capacidad de carga $K_{y}$ en (2), considerada constante, no es a menudo realista. Un entorno cambiante puede resultar en un cambio significativo en la capacidad de carga. Específicamente los cambios importantes en la ecología, el clima y el comportamiento humano favorecen el desarrollo de plagas urbanas que transmiten enfermedades [4]. Posterior a la emergencia de un vector, la falta de planificación y de vigilancia continua de los organismos de control vectorial, conlleva a la toma de medidas de emergencia para minimizar la proliferacion de los vectores, en cuyo caso, las campañas intentan vincular a la ciudadanía con algo imprescindible como la supresión permanente de los lugares de reproducción de los vectores en las viviendas y su proximidad, y el recobro de los valores de la austeridad a la hora de consumir [31].

Particularmente, se considera que las causas inmediatas que permiten que A. aegypti prospere en un territorio y aumente su potencial de transmisión de enfermedades al ser humano se relacionan directamente con las condiciones de saneamiento del peridomicilio y la persistencia de conductas y prácticas poco saludables, personales e institucionales. Estos factores acondicionan diariamente en el medio ambiente objetos que se pueden volver criaderos de mosquitos [23]. Nosotros suponemos que la reacción tardía de los ministerios de la Salud como las secretarías departamentales y municipales que no toman las medidas suficientes y oportunas para evitar la proliferación de los vectores, da lugar previamente a un periodo de crecimiento en forma aproximadamente exponencial de los criaderos.

Cuando el desorden ambiental impera, la población de vectores aumentan repentinamente, por ejemplo, porque los municipios carecen de servicios regulares 
de acueducto y esto favorece el almacenamiento de agua en condiciones inadecuadas, al igual que la disposición final de residuos sólidos, lo que aumenta el número de criaderos de mosquitos, y sufre una "explosión demográfica". Pero es más común que las poblaciones crezcan más despacio a tamaños más grandes, como si sufrieran una especie de "frenado". La causa de la inhibición podría ser la intensificación de las campañas de control entre la gente para frenar la proliferación de A. aegypti.

Entre dichas acciones de control, la importancia relativa de los criaderos se determina por la proporción de pupas que producen en un lugar determinado en un momento dado; esto puede servir como una aproximación para estimar la producción de mosquitos adultos [17]. Luego, la formulación de la capacidad de carga, $K_{y}$, varía directamente a la cantidad de criaderos en todas las categorías, $C_{i}$, con constante de proporcionalidad $\theta_{i}$. El recuento del número estimado de pupas en cada criadero, $\theta_{i}$, permite identificar las categorías de contenedores que producen la mayoría de los culícidos adultos.

Las intervenciones pueden ser dirigidas a controlar las crías en los contenedores más productivos, lo que a su vez, puede conducir a actividades focalizadas de control de vectores dirigidas a aquellos contenedores de mayor importancia epidemiológica, particularmente en áreas de alto riesgo de transmisión del dengue u otros virus [17] . La capacidad de carga de los estados inmaduros tiene la siguiente formulación:

$$
K_{y}(t)=\sum_{i=1}^{3} \theta_{i} C_{i}(t) .
$$

Proponemos entonces que el número promedio de los criaderos de cada categoría (baldes, tanques y llantas) en el entorno, en cualquier instante, variará a una determinada velocidad que dependerá o será función de dicha cantidad. Esta variación será cada vez más fuerte hasta un punto de inflexión y luego de eso amortiguada hasta alcanzar la máxima infestación a la que las cilivizaciones contribuyen. El primer periodo de mayor crecimiento simula las épocas de precipitaciones abundantes y temperaturas elevadas donde se suelen formar más criaderos y colectan la mayor cantidad de individuos de A. aegypti, en tanto que en la época de precipitaciones escasas y temperaturas bajas (estación seca), un segundo periodo describe incrementos paulatinamente más leves en la población y en los criaderos para A. aegypti.

Estas propiedades del crecimiento de los criaderos son emulación de la ley logística del crecimiento poblacional, matemáticamente expresado por las ecuaciones diferenciales (3), y por lo tanto, la dependencia funcional de $K_{y}(t)$ a $C_{i}(t)$ hace que $K_{y}(t)$ logísticamente también. 
La siguiente proposición establece que las soluciones del sistema (1)-(2), con condiciones iniciales en $\Xi \subset \Pi$, (4), permanecen en este subconjunto para todo tiempo futuro, consistente con el significado físico que se le otorga a los modelos de poblaciones.

$$
\Xi=\left\{(x, y) \in \mathbb{R}_{+}^{2}: 0 \leq y \leq K_{y}, 0 \leq x \leq \frac{\omega}{\epsilon} K_{y}\right\} .
$$

Proposición 2.1 (Invarianza de $\Xi$.) El conjunto $\Xi$ contiene una región compacta y positivamente invariante para el sistema (1)-(2)

Demostración. Supongamos que una solución $(x(t), y(t))$ con condición inicial $(x(0), y(0))$ interior a $\Xi$ conserva su positividad. Conocemos que la ecuación (3) es logística, por lo tanto, la variable $C_{i}(t)$ convergerá a $K_{i}$ para todo estado inicial en $t=0$ perteneciente al intervalo $0<C_{i}(0)<K_{i}$. La restricción $y(t) \leq K_{y} ; \forall t \in \mathbb{R}_{+}$y la segunda ecuación del sistema (1)-(2) implican que el extremo superior del recorrido de $y(t)$ es $\hat{K}_{y}=\theta_{1} K_{1}+\theta_{2} K_{2}+\theta_{3} K_{3}$, lo cual asegura a priori la acotación de $y(t) ; \forall t \in \mathbb{R}_{+}$.

Superiormente, la función $\varphi(\cdot) 2$ es acotada por la función lineal $\dot{x} \leq \omega \hat{K}_{y}-\epsilon x$ para cualquier solución de (1)-(2) iniciando en $\Xi$. Si desigualamos a cero y multiplicamos ambos lados por $e^{\epsilon t}$, obtenemos

$$
0 \geq\left(\dot{x}-\omega \hat{K}_{y}+\epsilon x\right) e^{\epsilon t}=\frac{d}{d t}\left[\left(\epsilon x-\omega \hat{K}_{y}\right) e^{\epsilon t} / \epsilon\right] .
$$

donde la función $\left(\epsilon x-\omega \hat{K}_{y}\right) e^{\epsilon t} / \epsilon$ tiene derivada negativa o cero y en ese orden es no creciente para $0 \leq t<+\infty$. Integrando desde 0 hasta $t$ da

$$
\frac{1}{\epsilon}\left(\epsilon x(t)-\omega \hat{K}_{y}\right) e^{\epsilon t} \leq \frac{1}{\epsilon}\left(\epsilon x(0)-\omega \hat{K}_{y}\right)
$$

Entonces,

$$
x(t) \leq \omega \hat{K}_{y} / \epsilon+\left(x_{0}-\omega \hat{K}_{y} / \epsilon\right) e^{-\epsilon t}
$$

y $x(t) \leq \omega \hat{K}_{y} / \epsilon$ si $t \longrightarrow+\infty$.

Ahora se demuestra que el campo vectorial $\mathbf{K}(x, y)=\varphi_{1}(x, y) \mathbf{i}+\varphi_{2}(x, y) \mathbf{j}$ cuyo dominio es la frontera de $\Xi$ apunta hacia su interior. 
De este modo, toda trayectoria $(x(t), y(t))$ de (1)-(2) permanece en $\Xi$ para todo $t \in \mathbb{R}_{+}$. Se define la frontera de $\Xi$, denotada $\partial \Xi$, como $\partial \Xi=\bigcup_{i=1}^{4} \Xi_{i}$ donde

$$
\begin{aligned}
& \partial \Xi_{1}=\left\{(x, y) \in \mathbb{R}^{2}: 0 \leq x \leq \frac{\omega}{\epsilon} \hat{K}_{y}, y=0\right\}, \\
& \partial \Xi_{2}=\left\{(x, y) \in \mathbb{R}^{2}: x=0,0 \leq y \leq \hat{K}_{y}\right\}, \\
& \partial \Xi_{3}=\left\{(x, y) \in \mathbb{R}^{2}: 0 \leq x \leq \frac{\omega}{\epsilon} \hat{K}_{y}, y=\hat{K}_{y}\right\}, \\
& \partial \Xi_{4}=\left\{(x, y) \in \mathbb{R}^{2}: x=\frac{\omega}{\epsilon} K_{y}, 0 \leq y \leq \hat{K}_{y}\right\} .
\end{aligned}
$$

K asocia a cada punto $P=\left(x_{0}, y_{0}\right)$ de $\partial \Xi$ el vector $\mathbf{w}=\varphi_{1}(P) \mathbf{i}+\varphi_{2}(P) \mathbf{j}$. Sea $\mathbf{v}$ el vector con punto inicial $P$ y punto terminal $T=\left(x_{0}+\varphi_{1}(P), y_{0}+\varphi_{2}(P)\right)$. Luego, para cada $P \in \partial \Xi$ :

(a) $P=\left(x_{0}, 0\right) \in \partial \Xi_{1} \Rightarrow T=\left(x_{0}(1-\epsilon), x_{0} f \phi\right) \therefore \exists \mathbf{v}^{\prime} \in \mathbb{R}^{2} \mid \mathbf{v}^{\prime}=\kappa \mathbf{v} \in \Xi$, $\kappa>0 \because 0 \leq x_{0}, 0<\phi, 0<f<1$.

(b) $P=\left(0, y_{0}\right) \in \partial \Xi_{2} \Rightarrow T=\left(y_{0} \omega, y_{0}(1-\omega-\pi)\right) \therefore \exists \mathbf{v}^{\prime} \in \mathbb{R}^{2}$ $\mid \mathbf{v}^{\prime}=\kappa \mathbf{v} \in \Xi, \kappa>0 \because 0 \leq y_{0}, 0<\omega$.

(c) $P=\left(x_{0}, \hat{K}_{y}\right) \in \partial \Xi_{3} \Rightarrow T=\left(\omega \hat{K}_{y}+x_{0}(1-\epsilon), \hat{K}_{y}(1-\omega-\pi)\right) \therefore$ $\exists \mathbf{v}^{\prime} \in \mathbb{R}^{2} \mid \mathbf{v}^{\prime}=\kappa \mathbf{v} \in \Xi, \kappa>0 \because(1-\omega-\pi) \hat{K}_{y}<(1-0) \hat{K}_{y}$.

(d) $P=\left(\frac{\omega}{\epsilon} \hat{K}_{y}, y_{0}\right) \in \partial \Xi_{4} \Rightarrow T=\left(\frac{\omega}{\epsilon} \hat{K}_{y}+\omega\left(y_{0}-\hat{K}_{y}\right), \frac{f \phi \omega}{\epsilon}\left(\hat{K}_{y}-y_{0}\right)+\right.$ $\left.y_{0}(1-\omega-\pi)\right) \therefore \exists \mathbf{v}^{\prime} \in \mathbb{R}^{2} \mid \mathbf{v}^{\prime}=\kappa \mathbf{v} \in \Xi, \kappa>0 \because y_{0} \leq \hat{K}_{y}, \omega>0$.

\section{Análisis de estabilidad}

Las ecuaciones (3), entendidas como la razón de cambio en el número de criaderos tipo $i$ por unidad de variación de $t$, están desacopladas de las ecuaciones (1) y (2), interpretadas en relación con el ciclo vital del mosquito, ya que no dependen de las variables $x$ e $y$; al contrario, la presencia del término $K_{y}$ en la ecuación (2) acopla el sistema en una vía. Notamos también que los tres tipos de criaderos (baldes, tanques y neumáticos) tienen un crecimiento logístico definido por (3). La $i$-ésima solución de (3) sujeta a la condición inicial $C_{i}(0)=C_{i 0}$ es la función logística:

$$
C_{i}(t)=\frac{C_{i 0} K_{i}}{C_{i 0}+\left(K_{i}-C_{i 0}\right) e^{-r_{i} t}} \quad(i=1,2,3)
$$


donde $r_{i}=\alpha_{i}-\beta_{i}$ es la tasa de crecimiento intrínseca. En teoría, el crecimiento logístico supone que un ecosistema es capaz de sostener, como máximo, una cantidad determinada de individuos en una población. Por ello, cuando la densidad poblacional es lo suficientemente grande, los recursos naturales empiezan a agotarse debido a la competencia entre los organismos, lo que desacelera el ritmo de crecimiento e impone el tope máximo alrededor del cual la población dejará de crecer e incluso comienza su decrecimiento para mantenerse relativamente constante a lo largo del tiempo [25].

El concepto de capacidad de carga es uno de los supuestos del crecimiento logístico, frecuentemente empleado en el manejo de los seres vivos. En este modelo extrapolamos este concepto a objetos, los criaderos de mosquitos.

Pasando al límite en la función logística obtenemos:

$$
\lim _{t \rightarrow \infty} C_{i}(t)=K_{i}
$$

que se cumple si y sólo si $r_{i}>0$, es decir, se crearán nuevos criaderos en el área colonizada por $A$. aegypti. Las demás posibilidades son: si $r_{i}=0$, los criaderos son generados por la comunidad humana y después eliminados intencional o fortuitamente en igual proporción y el lote de criaderos permanece estático en el valor inicial $C_{i}(0)=C_{i 0}$; si $r_{i}<0$, los criaderos se alteran o eliminan en menores cantidades a su producción.

Con el fin de estudiar estabilidad, interesados en el caso $r_{i}>0$, comprendamos el comportamiento de las soluciones que parten de posiciones iniciales muy cercanas al punto de equilibrio. Consideremos condiciónes iniciales $C_{i}(0)=C_{i 0}$. La gráfica de la función $\varphi_{3}$ en (3) es una parábola cóncava hacia abajo, intersecando el eje de la variable independiente $C_{i}$, en los puntos $(0,0)$ y $\left(K_{i}, 0\right)$. Si $r_{i}>0,0<\lambda \ll K_{i}$ y $K_{i}-\lambda<C_{i 0}<K_{i}$ en el conjunto $\Xi$, se tendrá que $\dot{C}_{i}>0$. Así, la curva con ecuación (5) es estrictamente creciente y acotada superiormente por la asíntota horizontal $C_{i}=K_{i}$. Se concluye que el punto crítico $\left(K_{1}, K_{2}, K_{3}\right)$ es local y asintóticamente estable.

Proposición 3.1 (Estabilidad local del punto de equilibrio $Q$. .) Sea $r_{i}=\alpha_{i}-$ $\beta_{i}$ y $Q=\left(K_{1}, K_{2}, K_{3}\right)$. La solución estacionaria $Q$ es: (a) localmente asintóticamente estable si $r_{i}>0$, (b) estable si $r_{i}=0, y(c)$ inestable si $r_{i}<0$.

Esta deducción del comportamiento a largo plazo del sistema dado en (3), comprobada la existencia del límite en el infinito de sus solución, nos permite reformular la ecuación (2), asumiendo una capacidad máxima de los estadios inmaduros (huevos, larvas y pupas) para las tres categorías de criaderos (baldes, tanques y neumáticos), y de paso, el descoplamiento del sistema en dos vías. 
Reemplazando $K_{y}=\hat{K}_{y}=\sum_{i=1}^{3} K_{i} \theta_{i}$ en (2), el sistema (1)-(3) se desacopla en el sistema subyacente:

$$
\begin{aligned}
& \dot{x}=\omega y-\epsilon x \equiv \varphi_{1}(x, y) \\
& \dot{y}=f \phi x\left(1-\frac{y}{\hat{K}_{y}}\right)-(\pi+\omega) y \equiv \varphi_{2}(x, y)
\end{aligned}
$$

el cual tiene trayectorias en la región de interés biológico:

$$
\Xi=\left\{(x, y) \in \mathbb{R}_{+}^{2}: 0 \leq x \leq \frac{\omega}{\epsilon} \sum_{i=1}^{3} K_{i} \theta_{i}, 0 \leq y \leq \sum_{i=1}^{3} K_{i} \theta_{i}\right\}
$$

Entretanto, las soluciones estacionarias del sistema (6)-(7) provienen de resolver el sistema algebraico no lineal $\varphi_{1}(x, y)=0, \varphi_{2}(x, y)=0$ para $x$ e $y$. Una solución inmediata es el equilibrio trivial, $x=y=0$. El equilibrio de coexistencia se determina despejando $x$ de $\varphi_{1}(x, y)=0$, esto es, $x=\frac{\omega}{\epsilon} y$; dicho valor se reemplaza en $\varphi_{2}(x, y)=0$ lo que permite hallar la otra raíz, $y=\left(1-\frac{\epsilon(\pi+\omega)}{f \phi \omega}\right) K_{y}$. Por ende, los puntos de equilibrio del sistema (6)-(7) son:

$$
P_{0}=(0,0), P_{1}=(\hat{x}, \hat{y})=\left(\frac{\omega}{\epsilon} \hat{y},\left(1-\mathrm{R}_{m}^{-1}\right) \sum_{i=1}^{3} K_{i} \theta_{i}\right)
$$

donde,

$$
\mathrm{R}_{m}=\frac{f \phi \omega}{\epsilon(\pi+\omega)}
$$

es denominado umbral de crecimiento poblacional del mosquito.

A grandes rasgos, el umbral (9) indica el número promedio de mosquitos hembras engendrados por un mosquito hembra y es determinante a la hora de establecer si la población continúa aumentando o en su lugar se elimina del entorno. Los términos que lo componen son: el número promedio de huevos hembras producidos por un mosquito hembra durante el tiempo esperado de vida de un mosquito hembra en su etapa adulta, $\frac{f \phi}{\epsilon}$, y la probabilidad de que mosquitos hembras que sobreviven al estadio inmaduro lleguen a la adultez, $\frac{\omega}{\pi+\omega}$. 
A continuación linealizamos el sistema (6)-(7), expandiendo las funciones $\varphi_{1}(\cdot)=\dot{x} \mathrm{y} \varphi_{2}(\cdot)=\dot{y}$ en series de Taylor en la vecindad de la solución constante $\left(x^{*}, y^{*}\right): x=x^{*}+u, y=y^{*}+v$. Obteniendo el sistema lineal en $u$ y $v$ :

$$
\begin{aligned}
& \frac{d u}{d t}=-\epsilon u+\omega v \\
& \frac{d v}{d t}=f \phi\left(1-\frac{\tilde{y}}{K_{y}}\right) u-\left(f \phi \frac{\tilde{x}}{K_{y}}+(\pi+\omega)\right) v .
\end{aligned}
$$

En forma matricial: $\dot{\mathbf{S}}=\mathbf{J S}$, donde

$$
\mathbf{S}=\left(\begin{array}{l}
u \\
v
\end{array}\right) \quad \text { y } \mathbf{J}(\tilde{x}, \tilde{y})=\left(\begin{array}{c}
-\epsilon \\
f \phi\left(1-\frac{\tilde{y}}{K_{y}}\right)
\end{array} \quad-\left(f \phi \frac{\tilde{x}}{K_{y}}+(\pi+\omega)\right)\right),
$$

$\mathbf{J}$ es la matriz jacobiana o matriz de coeficientes del sistema linealizado.

Las proposiciones de la 3.2 a 3.7 enunciadas en el resto de la presente sección son resultado del estudio de la estabilidad (local y global) de los puntos fijos de extinción y coexistencia de la población de mosquitos para el sistema (6)-(7).

\section{Proposición 3.2 (Estabilidad local del punto de equilibrio $P_{0}$.)}

Sea $\mathrm{R}_{m}=\frac{f \phi \omega}{\epsilon(\pi+\omega)}$. La solución estacionaria $P_{0}$ es $($ a) localmente asintóticamente estable si $\mathrm{R}_{m}<1$ (b) no hiperbólica si $\mathrm{R}_{m}=1$ y (c) inestable si $\mathrm{R}_{m}>1$.

Demostración. Las técnicas de análisis que aplicaremos pueden consultarse en [32]. La matriz jacobiana evaluada en la solución estacionaria $P_{0}=(0,0)$ toma la forma:

$$
\mathbf{J}(0,0)=\left(\begin{array}{cc}
-\epsilon & \omega \\
f \phi & -(\pi+\omega)
\end{array}\right)
$$

Su traza y determinante son $\operatorname{tr} \mathbf{J}(0,0)=-(\epsilon+\pi+\omega)$ y $\operatorname{det} \mathbf{J}(0,0)=\epsilon(\pi+\omega)\left(1-\mathrm{R}_{m}\right)$.

(a) $\mathrm{R}_{m}<1 \Rightarrow \operatorname{tr} \mathbf{J}(0,0)<0 \wedge \operatorname{det} \mathbf{J}(0,0)>0$. Por lo tanto, en virtud del criterio traza-determinante para sistemas bidimensionales, $P_{0}$ es localmente asinóticamente estable (un nodo).

(b) Con la ecuación característica $\operatorname{det}(\mathbf{J}(0,0)-\mu \mathbf{I})=0$ ó $\mu^{2}+\operatorname{tr} \mathbf{J}(0,0) \mu+$ $\operatorname{det} \mathbf{J}(0,0)=0$ hallamos que los valores propios son $\mu_{0}=0$ y $\mu_{1}=$ $-(\epsilon+\pi+\omega)$, cuando $\mathrm{R}_{m}=1$. Como $\Re\left(\mu_{0}\right)=0$, el equilibrio $P_{0}$ es no hiperbólico. 
(c) $\mathrm{R}_{m}>1 \Rightarrow \operatorname{det} \mathbf{J}(0,0)<0$. Por lo tanto, $P_{0}$ es inestable.

El origen es un punto fijo para (6)-(7) y la pregunta que hacemos es si es o no estable. Por lo tanto, dado que $P_{0}$ es no hiperbólico según el inciso $(b)$ de la Proposición 3.2, no podemos sacar conclusiones concernientes a la estabilidad o inestabilidad de este equilibrio sobre la base de la linealización. Responderemos a la pregunta de la estabilidad usando la teoría de la variedad central [39].

Proposición 3.3 (Variedad central local del punto de equilibrio $P_{0}$.) $\mathrm{R}_{m}=1$ implica que el equilibrio trivial del sistema (6)-(7) es estable.

Demostración. Los valores propios de (6)-(7) linealizado alrededor de $(x, y)=(0,0)$ son $\mu_{0}=0$ y $\mu_{1}=-(\epsilon+\pi+\omega)$ con la restricción $\mathrm{R}_{m}=1$. Para encontrar los subespacios estable $E^{\mathrm{s}}$ y central $E^{\mathrm{c}}$, basta resolver los sistemas homogéneos $\operatorname{det}\left(\mathbf{J}(0,0)-\mu_{0} \mathbf{I}\right)=0 \mathrm{y} \operatorname{det}\left(\mathbf{J}(0,0)-\mu_{1} \mathbf{I}\right)=0$. Las soluciones no triviales de estos sistemas son los vectores propios asociados a $\mu_{0}$ y $\mu_{1}$ que generan dichos subespacios:

$$
E^{\mathrm{s}}=\operatorname{gen}\left\{\left(\begin{array}{c}
1 \\
\frac{\epsilon}{\omega}
\end{array}\right)\right\} \text { y } E^{\mathrm{c}}=\operatorname{gen}\left\{\left(\begin{array}{c}
1 \\
\frac{\epsilon+\pi}{-\pi}
\end{array}\right)\right\}
$$

donde $\operatorname{dim} E^{\mathrm{s}}+\operatorname{dim} E^{\mathrm{c}}=2$.

Primero, debemos poner (10) en la forma estándar

$$
\left(\begin{array}{c}
\dot{\psi} \\
\dot{z}
\end{array}\right)=\left(\begin{array}{cc}
A_{1} & 0 \\
0 & A_{2}
\end{array}\right)\left(\begin{array}{l}
\psi \\
z
\end{array}\right)+\left(\begin{array}{l}
f_{1}(\psi, z) \\
f_{2}(\psi, z)
\end{array}\right) ; f_{i}(0,0)=D f_{i}(0,0)=0, i=1,2 .
$$

Usando las bases propias de los subespacios, obtenemos la transformación

$$
\left(\begin{array}{l}
x \\
y
\end{array}\right)=\left(\begin{array}{cc}
1 & 1 \\
\frac{\epsilon}{\omega} & -\frac{\pi+\omega}{\omega}
\end{array}\right)\left(\begin{array}{l}
\psi \\
z
\end{array}\right)
$$

con inversa

$$
\left(\begin{array}{l}
\psi \\
z
\end{array}\right)=\frac{1}{\epsilon+\pi+\omega}\left(\begin{array}{cc}
\pi+\omega & \omega \\
\epsilon & -\omega
\end{array}\right)\left(\begin{array}{l}
x \\
y
\end{array}\right)
$$

que transforma (6)-(7) en

$$
\begin{aligned}
\left(\begin{array}{c}
\dot{\psi} \\
\dot{z}
\end{array}\right)= & -z\left(\begin{array}{c}
0 \\
\epsilon+\pi+\omega
\end{array}\right)-\frac{f \phi(\epsilon-\pi-\omega)}{K_{y}(\epsilon+\pi+\omega)}\left(\begin{array}{r}
\psi z \\
-\psi z
\end{array}\right) \\
& -\frac{f \phi K_{y}^{-1}}{\epsilon+\pi+\omega}\left(\begin{array}{rr}
\epsilon & -(\pi+\omega) \\
-\epsilon & \pi+\omega
\end{array}\right)\left(\begin{array}{l}
\psi^{2} \\
z^{2}
\end{array}\right) .
\end{aligned}
$$


Del teorema de existencia para las variedades centrales, existe una variedad central para (11) que puede ser representada localmente como

$$
W_{\text {loc }}^{\mathrm{c}}(0)=\left\{(\psi, z) \in \mathbb{R}^{\mathrm{c}} \times \mathbb{R}^{\mathrm{s}}\left|z=f_{3}(\psi),\right| \psi \mid<\delta, h(0)=0, D f_{3}(0)=0\right\}
$$

para $\delta$ suficientemente pequeño. Asumimos que $f_{3}(\psi)$ posee la forma $f_{3}(\psi)=\gamma \psi^{2}+\zeta \psi^{3}+\mathcal{O}(4)$ y lo sustituimos con (11) en $\dot{z}=D f_{3}(\psi) \dot{\psi}$,

$$
A_{2} f_{3}(\psi)+f_{2}\left(\psi, f_{3}(\psi)\right)=\left(2 \gamma \psi+3 \zeta \psi^{2}+\mathcal{O}(3)\right)\left[A_{1} w+f_{1}\left(\psi, f_{3}(\psi)\right)\right]
$$

donde: $A_{1}=0, A_{2}=-(\epsilon+\pi+\omega)$,

$$
\begin{aligned}
& f_{1}(\psi, z)=-\frac{f \phi}{K_{y}(\epsilon+\pi+\omega)}\left[\epsilon \psi^{2}+(\epsilon-\pi-\omega) \psi z-(\pi+\omega) z^{2}\right] \\
& f_{2}(\psi, z)=\frac{f \phi}{K_{y}(\epsilon+\pi+\omega)}\left[\epsilon \psi^{2}+(\epsilon-\pi-\omega) \psi z-(\pi+\omega) z^{2}\right] .
\end{aligned}
$$

Tras prescindir de los términos de grado mayor que 3 da

$$
\left[\gamma-\frac{f \phi \epsilon}{K_{y} A_{2}^{2}}\right] \psi^{2}-\left[\zeta-\frac{f \phi \gamma}{K_{y} A_{2}^{2}}(3 \epsilon-\pi-\omega)\right] \psi^{3}=0
$$

es decir, $\gamma=\frac{f \phi \epsilon}{K_{y} A_{2}^{2}}$ y $\zeta=\epsilon\left(\frac{f \phi}{K_{y} A_{2}^{2}}\right)^{2}(3 \epsilon-\pi-\omega)$, y por lo tanto tenemos

$$
z=\left(\frac{f \phi \epsilon}{K_{y} A_{2}^{2}}\right) \psi^{2}+\epsilon\left(\frac{f \phi}{K_{y} A_{2}^{2}}\right)^{2}(3 \epsilon-\pi-\omega) \psi^{3}+\mathcal{O}(4)
$$

Considerado la proyección del campo de vectores de (11) en $z=f_{3}(\psi)$ sobre $E^{\mathrm{c}}: \dot{\psi}=f_{1}\left(\psi, h_{3}(\psi)\right)$, es decir,

$$
\dot{\psi}=-\frac{f \phi \epsilon}{K_{y}(\epsilon+\pi+\omega)} \psi^{2}+\mathcal{O}(3)
$$

y despreciando términos de orden superior a dos, se deduce la familia de hipérbolas que gobierna el comportamiento de las trayectorias en la vecindad de la solución trivial del sistema (6)-(7) si $\mathrm{R}_{m}=1$ :

$$
\psi(t)=-\frac{K_{y}(\epsilon+\pi+\omega)}{(f \phi \epsilon) t+c}, c \in \mathbb{R} .
$$

Es claro que $\psi=0$ es un valor crítico de (12) e independientemente de $c \in \mathbb{R}$, $\psi(t) \longrightarrow 0(t \longrightarrow+\infty)$; entonces $(x(t), y(t)) \longrightarrow(0,0)(t \longrightarrow+\infty)$. 
Proposición 3.4 (Estabilidad local del punto de equilibrio $P_{1}$.)

Sea $\mathrm{R}_{m}=\frac{f \phi \omega}{\epsilon(\pi+\omega)}$. La solución estacionaria $P_{1}$ es (a) localmente asintóticamente estable si $\mathrm{R}_{m}>1$, (b) $P_{0}=P_{1}$ si $\mathrm{R}_{m}=1$ y (c) inestable si $\mathrm{R}_{m}<1$.

Demostración. La matriz jacobiana evaluada en la solución estacionaria $P_{1}=(\hat{x}, \hat{y})=\left(\frac{\omega}{\epsilon} \hat{y},\left(1-\frac{\epsilon(\pi+\omega)}{f \phi \omega}\right) \sum_{i=1}^{3} K_{i} \theta_{i}\right)$ toma la forma:

$$
\mathbf{J}(\hat{x}, \hat{y})=\left(\begin{array}{cc}
-\epsilon & \omega \\
\frac{\epsilon(\pi+\omega)}{\omega} & -\frac{f \phi \omega}{\epsilon}
\end{array}\right) .
$$

$\mathrm{Su}$ traza y determinante son $\operatorname{tr} \mathbf{J}(\hat{x}, \hat{y})=-(\epsilon+f \phi \omega / \epsilon)$ y $\operatorname{det} \mathbf{J}(\hat{x}, \hat{y})=\pi \epsilon\left(\mathrm{R}_{m}-1\right)$.

(a) $\mathrm{R}_{m}>1 \Rightarrow \operatorname{tr} \mathbf{J}(\hat{x}, \hat{y})<0 \wedge \operatorname{det} \mathbf{J}(\hat{x}, \hat{y})>0$. Por lo tanto, en virtud del criterio traza-determinante para sistemas bidimensionales, $P_{1}$ es localmente asintóticamente estable.

(b) $\mathrm{R}_{m}=1 \Rightarrow \hat{y}=(0) \sum_{i=1}^{3} K_{i} \theta_{i}=0 \wedge \hat{x}=\frac{\omega}{\epsilon}(0)=0 \therefore P_{0}=P_{1}$.

(c) $\mathrm{R}_{m}<1 \Rightarrow \operatorname{det} \mathbf{J}(\hat{x}, \hat{y})<0$. Por lo tanto, $P_{1}$ es inestable.

Proposición 3.5 (Órbitas del sistema (6)-(7).) El sistema (6)-(7) no presenta órbitas cerradas yaciendo enteramente en $\Xi$.

Demostración. Consideremos el campo vectorial de clase $\mathrm{C}^{1}(\Xi)$ :

$\mathbf{F}(\boldsymbol{x}, \boldsymbol{y})=(\omega y-\epsilon x) \mathbf{i}+\left[f \phi x\left(1-\frac{y}{K_{y}}\right)-(\pi+\omega) y\right] \mathbf{j}=h(x, y) \mathbf{i}+w(x, y) \mathbf{j}$.

La divergencia de este campo vectorial

$$
\operatorname{div} \mathbb{F}=\frac{\partial h}{\partial x}+\frac{\partial w}{\partial y}=-\left(\epsilon+f \phi \frac{x}{K_{y}}+(\pi+\omega)\right)<0,
$$

no es idénticamente cero y no cambia de signo en $\Xi$. Por el criterio de Bendixson [39], el sistema (6)-(7) no tiene órbitas cerradas en el interior de $\Xi$.

Una herramienta para estudiar la estabilidad global de un punto fijo, es el uso de funciones de Lyapunov, pero estas tienen cierta peculiaridad, no hay un método universal para encontrarlas, más bien se requiere de la experticia personal. Dicho esto, se recurre a la teoría formulada por Lyapunov [12] con el objetivo de construir una candidata a función de Lyapunov que gobierna la dinámica de (6)-(7) alrededor del equilibrio sin población. 
Proposición 3.6 (Función de Lyapunov para el punto de equilibrio $P_{0}$.)

Si $\mathrm{R}_{m} \leq 1$, el campo vectorial $L(x, y)=f \phi x+\epsilon y$ es una función de Lyapunov en $\Xi$ para el punto fijo $P_{0}$ del sistema (6)-(7).

Demostración. Sea $L \quad: \quad \Xi \longmapsto \mathbb{R}^{+}$una función $\mathrm{C}^{1}(\Xi)$ tal que $L(x, y)=\left(a^{\prime}, b^{\prime}\right) \cdot(x, y)$, con $\left(a^{\prime}, b^{\prime}\right) \in \mathbb{R}^{2}$ y $(x, y) \in \Xi$. Luego,

$$
L(x, y)=a^{\prime} x+b^{\prime} y
$$

Claramente $\forall\left(a^{\prime}, b^{\prime}\right) \in \mathbb{R}^{2}$, se tiene $L(0,0)=0$ y $L(a, b)>0$ si $(a, b) \neq(0,0)$, pero $a^{\prime}>0, b^{\prime}>0$. Además, debido a que $L_{t}(x, y)=a^{\prime} \dot{x}+b^{\prime} \dot{y}$, entonces

$$
\begin{aligned}
L_{t}(a, b) & =a^{\prime}(\omega b-\epsilon a)+b^{\prime}\left[f \phi a\left(1-K_{y}^{-1} b\right)-(\pi+\omega) b\right] \\
& =\left[f \phi b^{\prime}-\epsilon a^{\prime}\right] a+\left[\omega a^{\prime}-(\pi+\omega) b^{\prime}\right] b-\left(f \phi b^{\prime} K_{y}^{-1}\right) a b \\
& =\left[f \phi b^{\prime}-\epsilon a^{\prime}\right] a+(\pi+\omega) b^{\prime}\left[a^{\prime}\left(b^{\prime}\right)^{-1} \omega(\pi+\omega)^{-1}-1\right] b-\frac{f \phi b^{\prime} b}{K_{y}(a)^{-1}} .
\end{aligned}
$$

Para $L_{t}(a, b)<0$ se hace

$$
\frac{a^{\prime}}{b^{\prime}} \frac{\omega}{\pi+\omega} \leq 1 \quad \text { y } \quad f \phi b^{\prime}=\epsilon a^{\prime} .
$$

Si $\mathrm{R}_{m}=\frac{f \phi \omega}{\epsilon(\pi+\omega)} \leq 1$, no se satisfarán las relaciones (13) a menos que $a^{\prime}=f \phi$ y $b^{\prime}=\epsilon$. En consecuencia,

$$
L(x, y)=f \phi x+\epsilon y
$$

Proposición 3.7 (Estabilidad global del punto de equilibrio $P_{0}$.) $S i \mathrm{R}_{m} \leq 1$, el punto fijo $P_{0}$ del sistema (6)-(7) es globalmente asintóticamente estable en $\Xi$.

Demostración. $L(x, y)=f \phi x+\epsilon y$ satisface las condiciones de estabilidad de Lyapunov:

1. $L(0,0)=0$.

2. $L(x, y)>0$ si $(x, y) \neq(0,0)$.

3. $L_{t}(x, y) \leq 0$ en $\Xi-\{(0,0)\}$. Puesto que $P_{1}$ es localmente asintóticamente estable cuando $\mathrm{R}_{m} \leq 1$ y $L_{t}(x, y)=f \phi \dot{x}+\epsilon \dot{y}=f \phi(\omega y-\epsilon x)+$ $\epsilon\left[f \phi x\left(1-K_{y}^{-1} y\right)-(\pi+\omega) y\right]=\epsilon\left[(\pi+\omega)\left(\mathrm{R}_{m}-1\right)-f \phi K_{y}^{-1} x\right] y$. Para $\mathrm{R}_{m}=1$, sea 


$$
\Psi=\left\{(x, y) \in \mathbb{R}^{2} \mid L_{t}(x, y)=0\right\}=\left\{(x, y) \in \mathbb{R}^{2} \mid x=0 \vee y=0\right\}
$$

el conjunto donde la derivada y la primitiva se anulan.

Así, toda solución del sistema (6)-(7) que entra a $\Psi$, salvo $P_{0}$, saldrá de $\Psi$ al interior de $\Xi$, porque $\Psi \subset \partial \Xi$ (Proposición 2.1). En virtud del Principio de Invarianza de LaSalle [39], $P_{0}$ es globalmente asintóticamente estable en $\Xi$.

\section{Análisis de sensibilidad}

En esta sección, se presenta el análisis de sensibilidad local del umbral de crecimiento del mosquito y del estado estacionario no trivial en función de los parámetros que actúan en el planteamiento del modelo, con valores hipotéticos y calculados reportados en la Tabla 1 , en el caso que $\mathrm{R}_{m}>1\left(\mathrm{R}_{m} \approx 56.5595\right.$; $f=0.5)$. Las tasas de ovoposición, emergencia y mortalidad de mosquitos adultos han sido calculadas a partir de las funciones de la temperatura (14) a (16) ajustadas por Chen and Hsieh (2012) y la tasa de mortalidad del estado acuático, dada por (17), se obtuvo como promedio aritmético de las tasas de mortalidad diaria para los huevos, las larvas y las pupas tomadas de Trpis (1972), Otero et al. (2006) y Focks et al. (1993) referenciados por Simoy et al. (2015).

En su investigación, Chen and Hsieh (2012) recogieron lecturas semanales de la temperatura media en Kaohsiung (Taiwan) de 2001 a 2010 y eligieron cuatro parámetros entomológicos en el proceso de modelado, debido a la disponibilidad de los valores observados o experimentales de la revisión de la literatura, incluyendo la tasa de oviposición, la tasa de maduración de mosquitos preadultos, y la tasa de mortalidad de mosquitos adultos. Con base en estos datos, evaluaron diferentes funciones dependientes de la temperatura, hasta obtener las que mejor se ajustaban a datos [6].

Por su parte, basado en experimentos de laboratorio, Trpis (1972) estimó que la mortalidad diaria de los huevos es $m_{1}=0.01$ [día ${ }^{-1}$ ] para temperaturas absolutas en el rango $278^{\circ} \mathrm{K} \leq \Upsilon \leq 303^{\circ} \mathrm{K}$; Otero et al. (2006) aproximan como una función de la temperatura anual promedio, la tasa de mortalidad diaria natural de las larvas por la expresión $m_{2}(\Upsilon)=0.01+0.9725 \exp \left[\frac{278-\Upsilon}{2.703}\right]$, válido en el rango de $278^{\circ} \mathrm{K} \leq \Upsilon \leq 303^{\circ} \mathrm{K}$, y con esta misma ecuación aproximan la tasa de mortalidad diaria natural de las pupas, $m_{3}(\Upsilon)=m_{2}(\Upsilon)$, válido en el rango $278^{\circ} \mathrm{K} \leq \Upsilon \leq 303^{\circ} \mathrm{K}[16]$. 
Tabla 1: Condiciones iniciales y parámetros calculados, $i \in\{1,2,3\}$.

\begin{tabular}{|c|c|c|}
\hline Parámetro & Connotación & Valor \\
\hline$x(0)$ & $\begin{array}{l}\text { Número promedio inicial de mosquitos hem- } \\
\text { bras en la fase aérea }\end{array}$ & 4000000 \\
\hline$y(0)$ & $\begin{array}{l}\text { Número promedio inicial de mosquitos hem- } \\
\text { bras en la fase acuática }\end{array}$ & 2000000 \\
\hline$\omega$ & $\begin{array}{l}\text { Tasa de desarrollo del estadio inmaduro al es- } \\
\text { tadio adulto }\end{array}$ & 0.0823 \\
\hline$\epsilon$ & Tasa de mortalidad del mosquito maduro & 0.0362 \\
\hline$\phi$ & Tasa de oviposición & 4.6472 \\
\hline$f$ & $\begin{array}{l}\text { Fracción de huevos que originan mosquitos } \\
\text { hembras }\end{array}$ & $0.0050 ; f^{*} ; 0.5000$ \\
\hline$\pi$ & Tasa de mortalidad de los estadios inmaduros & 0.0111 \\
\hline$r_{i}$ & $\begin{array}{l}\text { Tasa de crecimiento intrínseco de baldes }\left(r_{1}\right) \text {, } \\
\text { tanques }\left(r_{2}\right) \text { y llantas }\left(r_{3}\right)\end{array}$ & 0.5000 \\
\hline$\theta_{i}$ & $\begin{array}{l}\text { Número promedio de huevos, larvas y pupas } \\
\text { alojados en baldes }\left(\theta_{1}\right) \text {, tanques }\left(\theta_{2}\right) \text { y llantas } \\
\left(\theta_{3}\right)\end{array}$ & $\begin{array}{l}250 ; 500 ; 750 \\
\text { respectivamente }\end{array}$ \\
\hline$K_{i}$ & $\begin{array}{l}\text { Número máximo de criaderos de la categoría } \\
\text { baldes }\left(K_{1}\right) \text {, tanques }\left(K_{2}\right) \text { o llantas }\left(K_{3}\right) \text { en } \\
\text { el medio }\end{array}$ & $\begin{array}{l}1000 ; 2000 ; 3000 \\
\text { respectivamente }\end{array}$ \\
\hline$K_{y}$ & $\begin{array}{l}\text { Número max́imo de huevos-larvas-pupas en } \\
\text { el medio }\end{array}$ & 3500000 \\
\hline
\end{tabular}

En resumen, los parámetros dependientes de la temperatura en la escala de Celsius y en el rango $15^{\circ} \mathrm{C} \leq T \leq 29.85^{\circ} \mathrm{C}$ se definen en (14)-(17):

$$
\begin{aligned}
\phi(T)= & -0.0163 T^{2}+1.2897 T-15.837 \\
\omega(T)= & -0.000000338 T^{5}+0.00003909 T^{4}-0.00174765 T^{3} \\
& +0.037577293 T^{2}-0.378999315 T+1.426703347, \\
\epsilon(T)= & -0.000000232 T^{5}+0.00002943 T^{4}-0.00143848 T^{3} \\
& +0.033894016 T^{2}-0.385870020 T+1.739083020, \\
\pi(T)= & 0.01+0.6484 \exp \left(\frac{4.85-T}{2.703}\right) .
\end{aligned}
$$


Como contexto de aplicación, Armenia (Quindío) es una ciudad colombiana posicionada en la Región Andina cuya temperatura histórica anual ha oscilado entre $16^{\circ} \mathrm{C}$ y $28^{\circ} \mathrm{C}$, con una temperatura media anual de $22^{\circ} \mathrm{C}$ [14]. Los parámetros $\phi, \omega, \epsilon \mathrm{y} \pi$ se calcularon a una temperatura de $22^{\circ} \mathrm{C}$ y se reemplazaron en el umbral (9) para asignar el valor de la fracción $f$ cuando el umbral es menor, igual $\left(f=f^{*}\right)$ y mayor que la unidad.

Ahora para identificar los parámetros más significativos y sensibles en este modelo, se calcularon los índices de sensibilidad hacia adelante normalizados. Según [7], dada una variable $P$ diferenciable de un parámetro $\rho$, su índice de sensibilidad hacia adelante normalizado se define como:

$$
I_{\rho}^{P}=\frac{\partial P / P}{\partial \rho / \rho}=\frac{\partial P}{\partial \rho} \frac{\rho}{P}
$$

La sensibilidad local es una medida relativa del cambio en una salida del sistema cuando el valor de una entrada cambia, o la influencia de un parámetro en la variación de otro. Esto se logra realizando pequeñas variaciones en aquellos parámetros con el índice de sensibilidad de mayor magnitud [9].

A partir del umbral de crecimiento del mosquito (9), se obtiene los siguientes índices de sensibilidad de $\mathrm{R}_{m}$ con respecto a los parámetros $f, \phi, \omega, \epsilon \mathrm{y} \pi$ :

$$
\begin{aligned}
I_{f}^{\mathrm{R}_{m}} & =\frac{\partial \mathrm{R}_{m}}{\partial f} \frac{f}{\mathrm{R}_{m}}=\frac{\phi \omega}{\epsilon(\pi+\omega)} \frac{f}{\mathrm{R}_{m}}=1, \\
I_{\omega}^{\mathrm{R}_{m}} & =\frac{\partial \mathrm{R}_{m}}{\partial \omega} \frac{\omega}{\mathrm{R}_{m}}=\frac{f \phi \pi}{\epsilon(\pi+\omega)^{2}} \frac{\omega}{\mathrm{R}_{m}}=\frac{\pi}{\pi+\omega}, \\
I_{\phi}^{\mathrm{R}_{m}} & =\frac{\partial \mathrm{R}_{m}}{\partial \phi} \frac{\phi}{\mathrm{R}_{m}}=\frac{f \omega}{\epsilon(\pi+\omega)} \frac{\phi}{\mathrm{R}_{m}}=1, \\
I_{\epsilon}^{\mathrm{R}_{m}} & =\frac{\partial \mathrm{R}_{m}}{\partial \epsilon} \frac{\epsilon}{\mathrm{R}_{m}}=-\frac{f \phi \omega}{\epsilon^{2}(\pi+\omega)} \frac{\epsilon}{\mathrm{R}_{m}}=-1, \\
I_{\pi}^{\mathrm{R}_{m}} & =\frac{\partial \mathrm{R}_{m}}{\partial \pi} \frac{\pi}{\mathrm{R}_{m}}=\frac{-f \phi \omega}{\epsilon(\pi+\omega)^{2}} \frac{\pi}{\mathrm{R}_{m}}=-I_{\omega}^{\mathrm{R}_{m}} .
\end{aligned}
$$

Los parámetros de alta sensibilidad para el umbral de crecimiento del mosquito son $f$ y $\phi$ por encima de $\omega$ y $\pi$, siendo menos sensible a $\epsilon$. La Tabla 2 muestra los índices de sensibilidad para $\mathrm{R}_{m}$, tomando tres cifras decimales. 
Tabla 2: Índices de sensibilidad de $\mathrm{R}_{m}$.

\begin{tabular}{c|ccccc}
\hline \hline Parámetro $(\rho)$ & $f$ & $\phi$ & $\omega$ & $\epsilon$ & $\pi$ \\
\hline Índice $\left(I_{\rho}^{\mathrm{R}_{m}}\right)$ & 1 & 1 & 0.118 & -1 & -0.118 \\
\hline \hline
\end{tabular}

Los índices anteriores sugieren que se puede controlar el tamaño poblacional de A. aegypti reduciendo cuidadosamente alguno de los parámetros más sensibles (fracción de huevos con embrión hembra y tasa de ovoposición), o bien, aumentar el parámetro menos sensible (la tasa de mortalidad del mosquito maduro).

Mediante el punto de equilibrio $P_{1}$ dado en (8), se tiene que los índices de sensibilidad de $\hat{x}$ con respecto a $f, \phi, \omega, \epsilon, \pi, \theta_{j}$ y $K_{j}(j=1,2,3)$ son:

$$
\begin{aligned}
I_{f}^{\hat{x}} & =\frac{\partial \hat{x}}{\partial f} \frac{f}{\hat{x}}=\frac{f}{\hat{x}} \frac{\pi+\omega}{f^{2} \phi} \sum_{i=1}^{3} \theta_{i} K_{i}=\frac{1}{\mathrm{R}_{m}-1}, \\
I_{\epsilon}^{\hat{x}} & =\frac{\partial \hat{x}}{\partial \epsilon} \frac{\epsilon}{\hat{x}}=-\frac{\epsilon}{\hat{x}} \frac{\omega}{\epsilon^{2}} \sum_{i=1}^{3} \theta_{i} K_{i}=-\mathrm{R}_{m} I_{f}^{\hat{x}}, \\
I_{\phi}^{\hat{x}} & =\frac{\partial \hat{x}}{\partial \phi} \frac{\phi}{\hat{x}}=\frac{\phi}{\hat{x}} \frac{\pi+\omega}{f \phi^{2}} \sum_{i=1}^{3} \theta_{i} K_{i}=I_{f}^{\hat{x}}, \\
I_{\pi}^{\hat{x}} & =\frac{\partial \hat{x}}{\partial \pi} \frac{\pi}{\hat{x}}=-\frac{\pi}{\hat{x}} \frac{1}{f \phi} \sum_{i=1}^{3} \theta_{i} K_{i}=-I_{\omega}^{\mathrm{R}} I_{f}^{\hat{x}}, \\
I_{\theta_{j}}^{\hat{x}} & =\frac{\partial \hat{x}}{\partial \theta_{j}} \frac{\theta_{j}}{\hat{x}}=\theta_{j} K_{j}\left(\sum_{i=1}^{3} \theta_{i} K_{i}\right)^{-1}, \\
I_{K_{j}}^{\hat{x}} & =\frac{\partial \hat{x}}{\partial K_{j}} \frac{K_{j}}{\hat{x}}=I_{\theta_{j}}^{\hat{x}}, \\
I_{\omega}^{\hat{x}} & =\frac{\partial \hat{x}}{\partial \omega} \frac{\omega}{\hat{x}}=\frac{\omega}{\hat{x}} \frac{f \phi-\epsilon}{f \phi \epsilon} \sum_{i=1}^{3} \theta_{i} K_{i}=\frac{I_{f}^{\hat{x}} \mathrm{R}_{m}}{f \phi}(f \phi-\epsilon) .
\end{aligned}
$$

La Tabla 3 muestra los índices de sensibilidad de $\hat{x}$ hasta tres cifras decimales. Se observa que los parámetros más sensibles para la población de mosquitos hembras maduros son $\omega, \theta_{1}, \theta_{2}, \theta_{3}, K_{1}, K_{2}$ y $K_{3}$, contrario a lo sucede $\operatorname{con} \pi$ y $\epsilon$.

Con base en la Tabla 3 estrategias como el incremento racional de las tasas de mortalidad de los mosquitos maduros e inmaduros $\pi$ y $\epsilon$ (parámetros de menor sensibilidad), o la disminución de la tasa de maduración de los estados 
Tabla 3: Índices de sensibilidad para $\hat{x}$.

\begin{tabular}{c|cccccc}
\hline \hline Parámetro $(\rho)$ & $f$ & $\phi$ & $\omega$ & $\epsilon$ & $\pi$ & \\
\hline Índice $\left(I_{\rho}^{\hat{x}}\right)$ & 0.017 & 0.017 & 1.002 & -1.017 & -0.002 & \\
\hline \hline Parámetro $(\rho)$ & $\theta_{1}$ & $\theta_{2}$ & $\theta_{3}$ & $K_{1}$ & $K_{2}$ & $K_{3}$ \\
\hline Índice $\left(I_{\rho}^{\hat{x}}\right)$ & 0.071 & 0.285 & 1.642 & 0.071 & 0.285 & 1.642 \\
\hline \hline
\end{tabular}

inmaduros $\omega$, de las capacidades de carga de los criaderos y del número promedio de crías alojadas en estos recipientes (parámetros de mayor sensibilidad), son efectivas en el control de la densidad de los mosquitos.

Los índices de sensibilidad de $\hat{y}$ con respecto a los parámetros $f, \phi, \omega, \epsilon, \pi$, $\theta_{j}$ y $K_{j}(j=1,2,3)$ son:

$$
\begin{aligned}
I_{f}^{\hat{y}} & =\frac{\partial \hat{y}}{\partial f} \frac{f}{\hat{y}}=\frac{f}{\hat{y}} \frac{\epsilon(\pi+\omega)}{f^{2} \phi \omega} \sum_{i=1}^{3} \theta_{i} K_{i}=I_{f}^{\hat{x}}, \\
I_{\epsilon}^{\hat{y}} & =\frac{\partial \hat{y}}{\partial \epsilon} \frac{\epsilon}{\hat{x}}=-\frac{\epsilon}{\hat{y}} \frac{\pi+\omega}{f \phi \omega} \sum_{i=1}^{3} \theta_{i} K_{i}=-I_{f}^{\hat{x}}, \\
I_{\phi}^{\hat{y}} & =\frac{\partial \hat{y}}{\partial \phi} \frac{\phi}{\hat{y}}=\frac{\phi}{\hat{y}} \frac{\epsilon(\pi+\omega)}{f \phi^{2} \omega} \sum_{i=1}^{3} \theta_{i} K_{i}=I_{f}^{\hat{x}}, \\
I_{\pi}^{\hat{y}} & =\frac{\partial \hat{y}}{\partial \pi} \frac{\pi}{\hat{x}}=-\frac{\pi}{\hat{y}} \frac{\epsilon}{f \phi \omega} \sum_{i=1}^{3} \theta_{i} K_{i}=I_{\pi}^{\hat{x}}, \\
I_{\omega}^{\hat{y}} & =\frac{\partial \omega}{\partial \hat{y}} \frac{\omega}{\hat{y}}=\frac{\omega}{\hat{y}} \frac{\epsilon \pi}{f \phi \omega^{2}} \sum_{i=1}^{3} \theta_{i} K_{i}=-I_{\pi}^{\hat{x}}, \\
I_{\theta_{j}}^{\hat{y}} & =\frac{\partial \hat{y}}{\partial \theta_{j}} \frac{\theta_{j}}{\hat{y}}=I_{\theta_{j}}^{\hat{x}}, \\
I_{K_{j}}^{\hat{y}} & =\frac{\partial \hat{y}}{\partial K_{j}} \frac{K_{j}}{\hat{y}}=I_{\theta_{j}}^{\hat{x}} .
\end{aligned}
$$

La Tabla 4 muestra los índices de sensibilidad de $\hat{y}$ hasta milésimas. Se estima que los parámetros más sensibles para la población de mosquitos inmaduros son las capacidades de carga del medio ambiente a baldes, tanques y llantas, es decir $K_{1}, K_{2}$ y $K_{3}$, y los respectivos números promedios de estados inmaduros albergables, es decir $\theta_{1}, \theta_{2}$ y $\theta_{3}$. En cambio, los parámetros $\epsilon$ y $\pi$ ejercen menor influencia. 
Tabla 4: Índices de sensibilidad para $\hat{y}$.

\begin{tabular}{c|cccccc}
\hline \hline Parámetro $(\rho)$ & $f$ & $\phi$ & $\omega$ & $\epsilon$ & $\pi$ & \\
\hline Índice $\left(I_{\rho}^{\hat{y}}\right)$ & 0.017 & 0.017 & 0.002 & -0.017 & -0.002 & \\
\hline \hline Parámetro $(\rho)$ & $\theta_{1}$ & $\theta_{2}$ & $\theta_{3}$ & $K_{1}$ & $K_{2}$ & $K_{3}$ \\
\hline Índice $\left(I_{\rho}^{\hat{y}}\right)$ & 0.071 & 0.285 & 1.642 & 0.071 & 0.285 & 1.642 \\
\hline \hline
\end{tabular}

Esto sugiere que una política de control dirigida a reducir las capacidades de carga de criaderos potenciales, aumentar la tasa de mortalidad de las etapas acuáticas, o incrementar la tasa de mortalidad del mosquito aéreo, incurrirá en la disminución del tamaño de la población de mosquitos.

\section{Simulaciones numéricas}

Los simulaciones numéricas del umbral de crecimiento poblacional, las soluciones estacionarias y el plano de fase del sistema dinámico fueron efectuados usando el programa MAPLE con las condiciones iniciales y valores hipotéticos y calculados para los parámetros antes mencionados en la Tabla 1.

La Figura 1 muestra y compara soluciones particulares de la cantidad promedio instantánea de los criaderos (baldes, tanques y neumáticos) para las condiciones relativas de la tasa de crecimiento intrínseca. La Figuras 2, 3 y 4 presentan en tres escenarios el campo de pendientes y curvas solución para $x(t)$ y $y(t)$, correspondientes a $\mathrm{R}_{m}>1, \mathrm{R}_{m}=1$ y $\mathrm{R}_{m}<1$, que permiten visualizar en cada casos la estabilidad del punto de equilibrio. Finalmente, cada gráfica de las Figuras 5 y 6 es obtenida variando un parámetro y manteniendo fijos los demás parámetros del modelo.

Observamos que la negatividad de la tasa de crecimiento intrínseca (columna izquierda) provoca una tendencia de los criaderos a desaparecer del medio ambiente, es decir, los recipientes no útiles o en mal estado son destruidos o neutralizados para imponer barreras a la proliferación del vector e interrumpir el contacto entre las personas y los vectores. En cambio, cuando la $i$-ésima tasa de crecimiento intrínseca es positiva (columna derecha), cada trayectoria tiende a la capacidad de carga ambiental del $i$-ésimo criadero, lo suficientemente cercana al pasar el tiempo (véase la Figura 2(a-f)). 
(a)
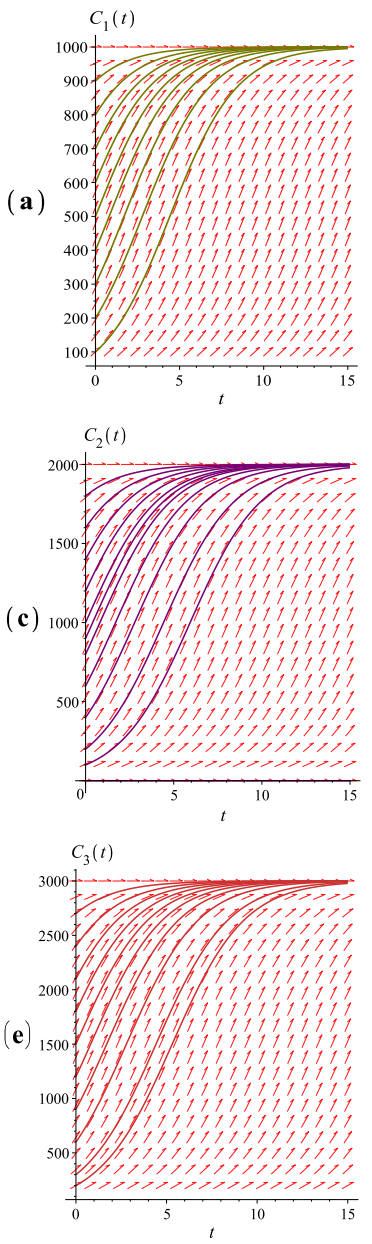

(b)

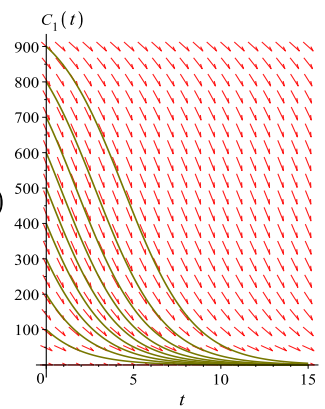

$C_{2}(t)$

(d)
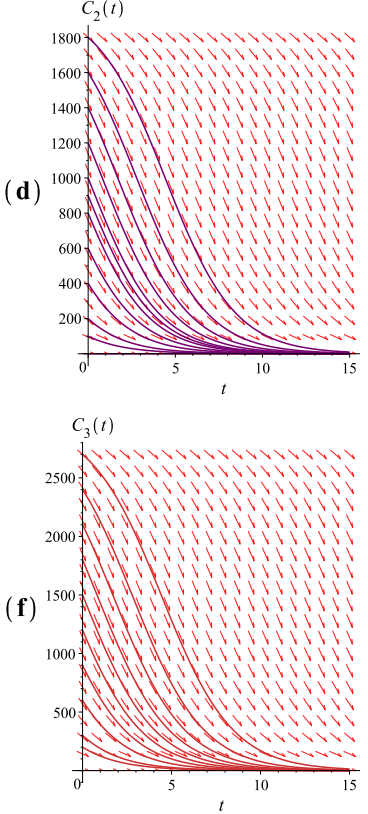

Figura 2: Comportamiento en el tiempo del volumen de baldes $C_{1}(t)$ (一), tanques $C_{2}(t)(-)$ y llantas $C_{3}(t)(-)$, con $r_{i}=0.5$ y $r_{i}=-0.5(i=1,2,3)$ en el mapa de fase.

Evidenciamos el comportamiento estable en el tiempo de la población de mosquitos adultos y de fases inmaduras. Con $\mathrm{R}_{m}>1$ el comportamiento cualitativo en el plano de fase explica la tendencia de las trayectorias hacia el punto de equilibrio no trivial $P_{1}=(3438118.293,7816495.456)$, el cual se comporta como un atractor (véase figura 3); con $\mathrm{R}_{m}<1$ el comportamiento geométrico en el plano de fase refleja la evolución de las órbitas hacia el estado estacionario trivial $P_{0}=(0,0)$, asintóticamente estable (véase Figura 4). 

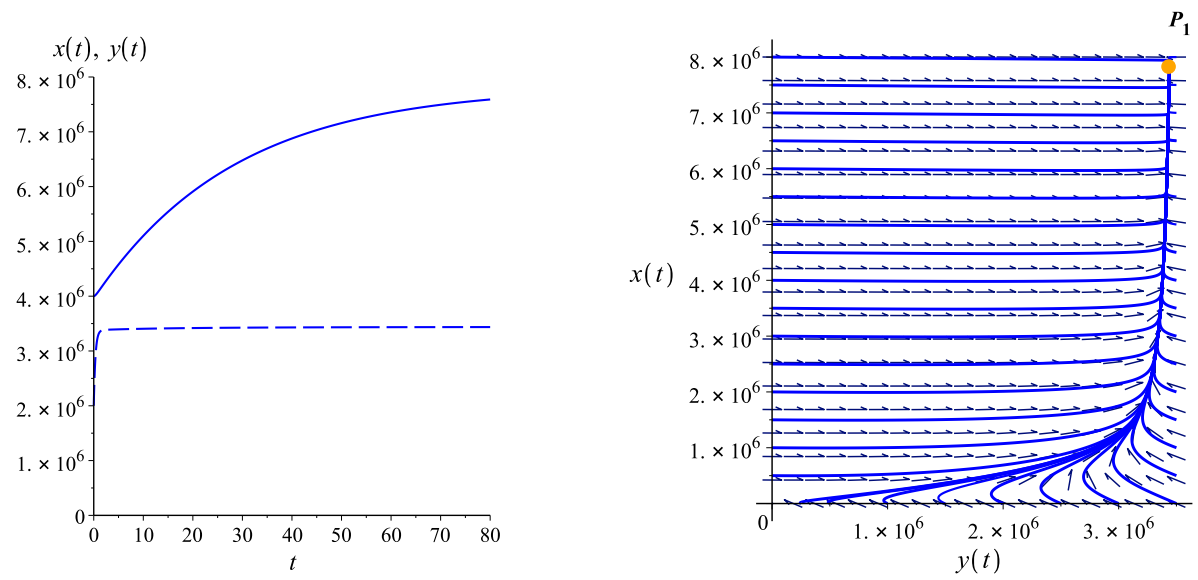

Figura 3: Comportamiento temporal de las poblaciones, mosquitos maduros $x(t)(-)$ y mosquitos inmaduros $y(t)(-\boldsymbol{-})$, con $\mathrm{R}_{m} \approx 56.5595$ y el respectivo plano de fase.
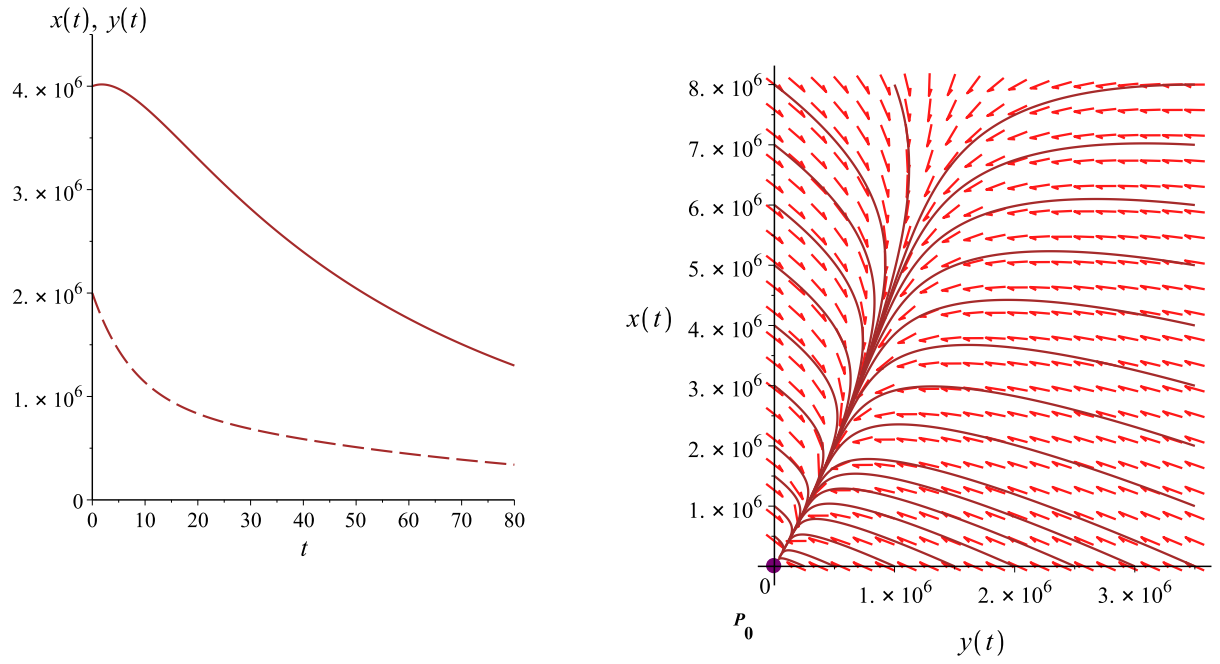

Figura 4: Comportamiento temporal de las poblaciones, mosquitos maduros $x(t)(-)$ y estadios inmaduros $y(t)\left({ }^{-}=\right)$, con $\mathrm{R}_{m} \approx 0.5655 \mathrm{y}$ el respectivo plano de fase.

Las gráficas de la Figura 5 muestran las soluciones del sistema con $\mathrm{R}_{m}=1$. Se detecta un decaimiento no exponencial hacia cero de las subpoblaciones 
adulta y preadulta del vector, a diferencia del escenario para un umbral de crecimiento menor que 1 , debido a que en este caso $P_{0}=(0,0)$ es estable pero no asintóticamente estable.
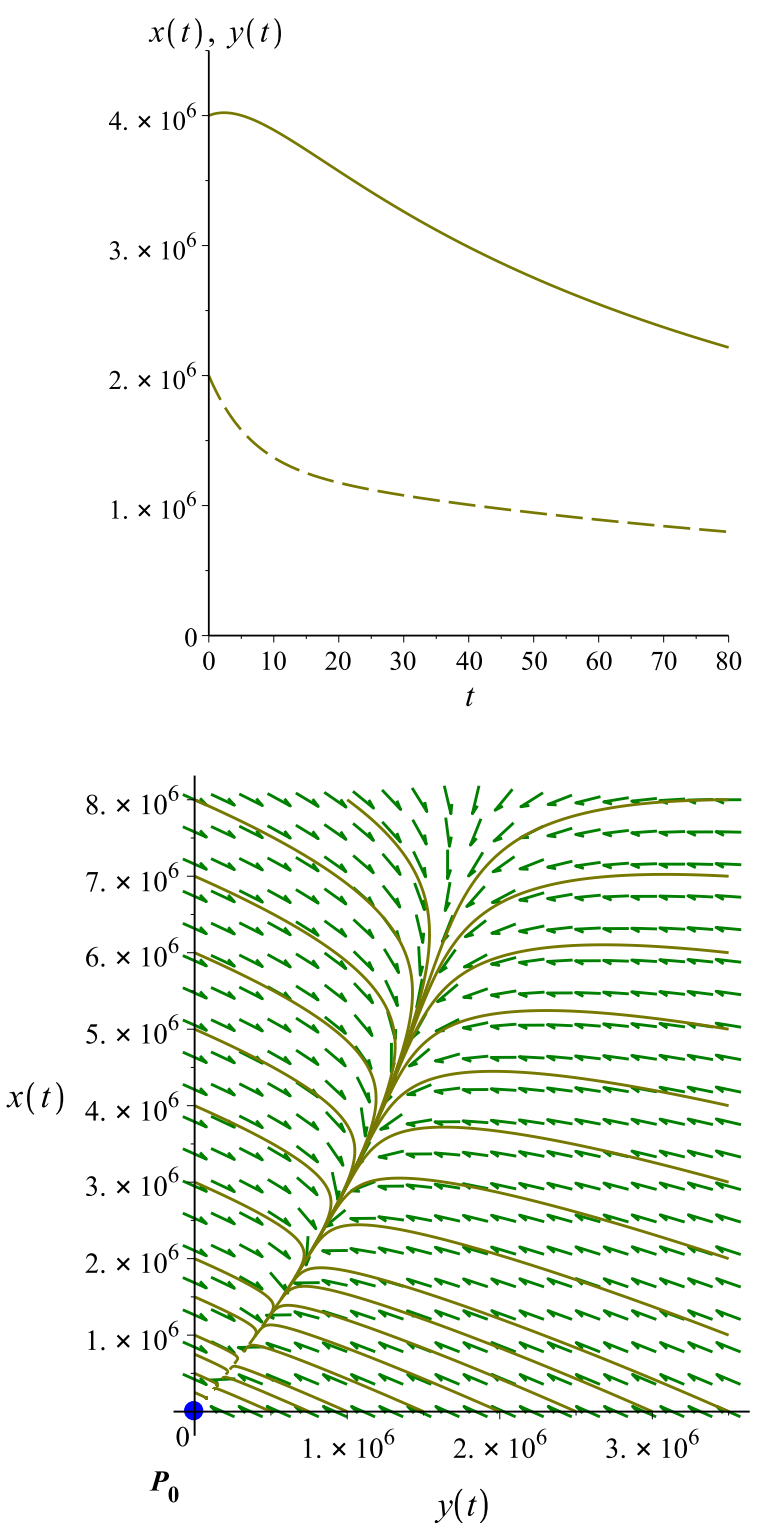

Figura 5: Comportamiento temporal de las poblaciones, mosquitos maduros $x(t)(-)$ y mosquitos inmaduros $y(t)\left(\boldsymbol{\Xi}^{-}\right)$, con $\mathrm{R}_{m}=1$ y el respectivo plano de fase. 
La Figura 6 contiene los gráficos del umbral de crecimiento del mosquito como función de cada uno de los parámetros. Observamos que $f, \phi$ y $\omega$ son directamente proporcionales a $\mathrm{R}_{m}$, a la vez que $\epsilon \mathrm{y} \pi$ son inversamente proporcionales al umbral.

$-\mathrm{R}_{m}(f)-\mathrm{R}_{m}(\phi)-\mathrm{R}_{m}(\omega)-\mathrm{R}_{m}(\epsilon)-\mathrm{R}_{m}(\pi)$

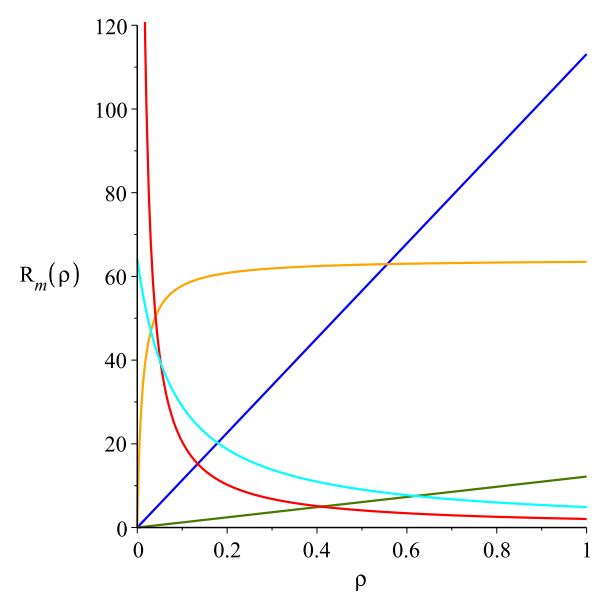

Figura 6: Umbral de crecimiento versus $\rho, \rho \in\{f, \phi, \omega, \epsilon, \pi\}$.

La Figura 7(a-c) relaciona a $\hat{x}$ con los parámetros $f, \phi, \omega, \epsilon, \pi, \theta_{1}, \theta_{2}, \theta_{3}$, $K_{1}, K_{2}$ y $K_{3}$. En ellos se ilustra que a medida que aumentan $f, \phi, \omega, \theta_{1}$, $\theta_{2}, \theta_{3}, K_{1}, K_{2}$ y $K_{3}$ aumenta el tamaño de la población de mosquitos hembras maduros, no obstante $\omega, \theta_{1}, \theta_{2}, \theta_{3}, K_{1}, K_{2}$ y $K_{3}$ hacen que se acelere todavía más el crecimiento de esta población. Por otro lado, a medida que aumentan los parámetros $\pi$ y $\epsilon$, disminuye la densidad poblacional en los mosquitos hembra adultos. 


$$
-\hat{x}(f)-\hat{x}(\phi)-\hat{x}(\omega)-\hat{x}(\epsilon)-\hat{x}(\pi)
$$
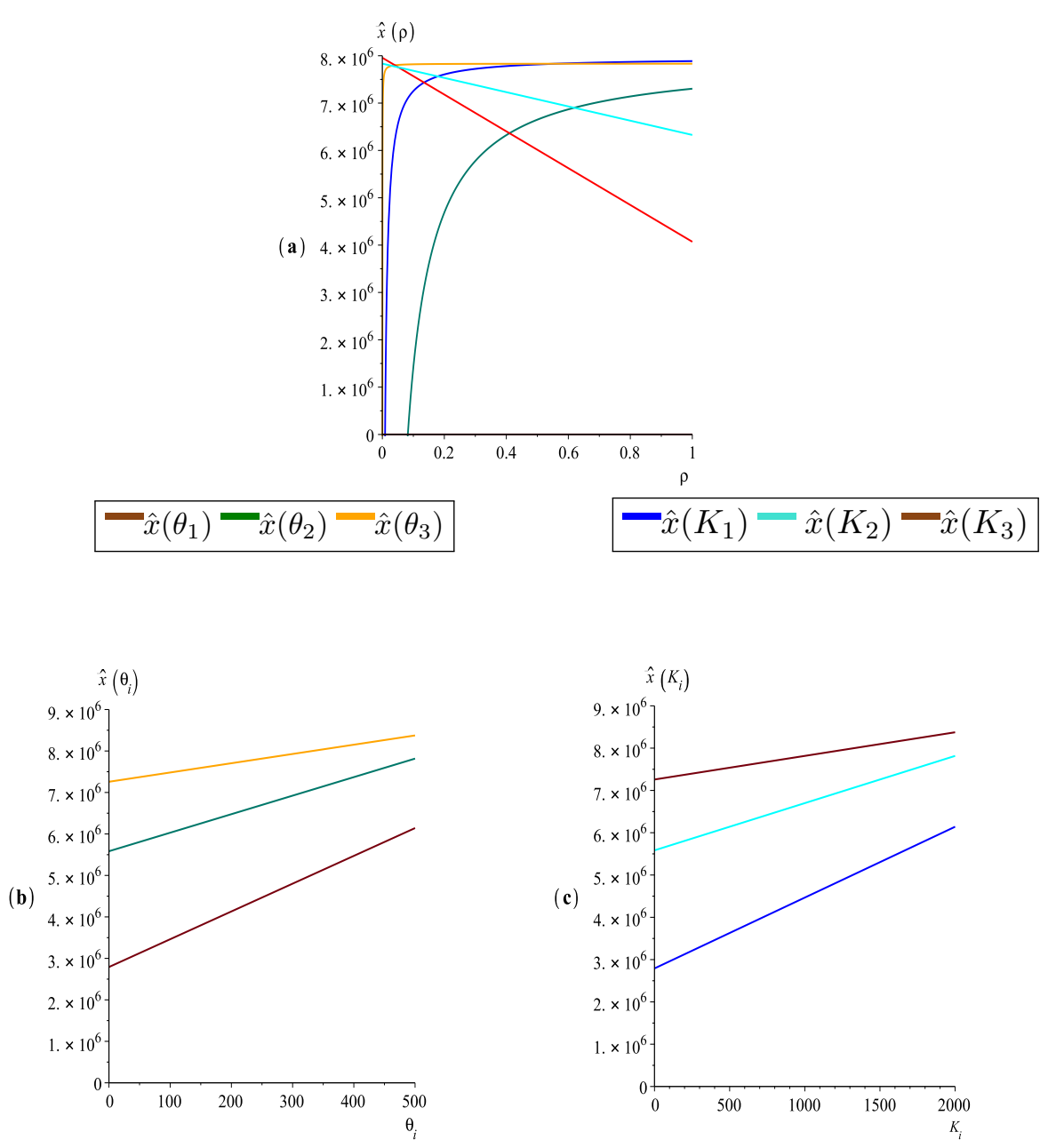

Figura 7: Componente $\hat{x}$ versus $\rho, \theta_{i}$ y $K_{i} ; \rho \in\{f, \phi, \omega, \epsilon, \pi\}$.

Análogamente, la figura 8(a-c) relaciona a $\hat{y} \operatorname{con}$ los parámetros $f, \phi, \omega, \epsilon$, $\pi, \theta_{1}, \theta_{2}, \theta_{3}, K_{1}, K_{2}$ y $K_{3}$. En ellos se observa que la variación del tamaño poblacional de los estados immaduros es directamente proporcional a $f, \phi, \omega$, $\theta_{1}, \theta_{2}, \theta_{3}, K_{1}, K_{2}$ y $K_{3}$, e inversamente proporcional a $\epsilon$ y $\pi$, siendo $\theta_{1}, \theta_{2}, \theta_{3}$, $K_{1}, K_{2}$ y $K_{3}$ los que estimulan aún más el crecimiento de esta población. 


$$
-\mathrm{R}_{m}(f)-\mathrm{R}_{m}(\phi)-\mathrm{R}_{m}(\omega)-\mathrm{R}_{m}(\epsilon)-\mathrm{R}_{m}(\pi)
$$

(a)

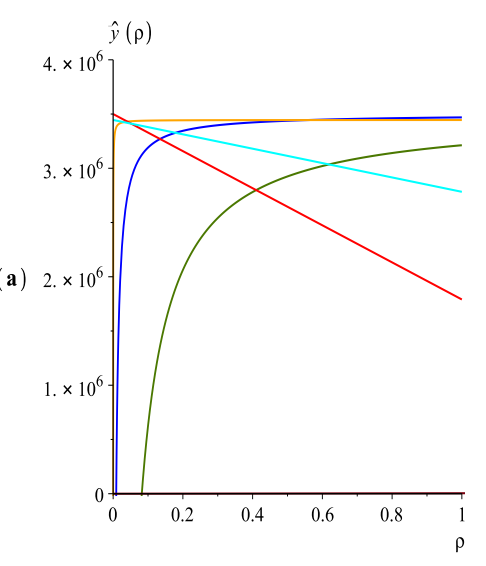

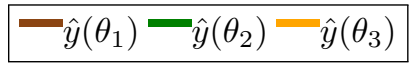

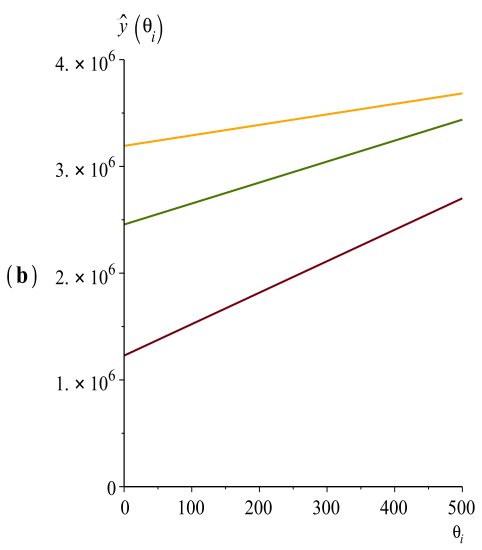

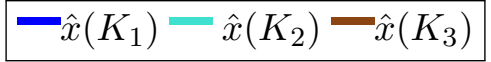

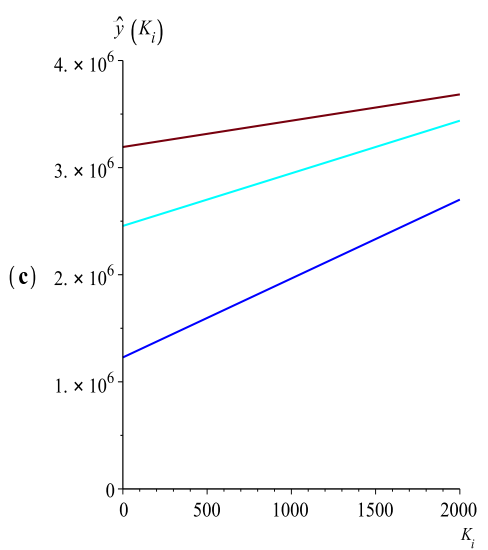

Figura 8: Componente $\hat{y}$ versus $\rho, \theta_{i}$ y $K_{i} ; \rho \in\{f, \phi, \omega, \epsilon, \pi\}$. 


\section{Discusión y resultados}

La literatura entomológica y epidemiológica consultada mostró que en algunos recipientes artificiales proliferan grandes cantidades de mosquitos inmaduros, mientras que otros resultan menos productivos. Por lo tanto, los esfuerzos por controlar a los vectores han de dirigirse a aquellos hábitats que sean más productivos y en consecuencia más importantes desde el punto de vista epidemiológico, en lugar de dirigirse a todo tipo de recipientes, especialmente cuando existan notables limitaciones de recursos [2].

La gestión ambiental es un método congruente ecológicamente, porque no conlleva el exterminio total de la población vectora y efectos secundarios para el ecosistema, pues actúa sobre los factores de riesgo que facilitan la infestación del vector, entre los que se destacan la urbanización no controlada ni planificada, la falta de infraestructura sanitaria adecuada, sin suministro de agua continua, servicios de recogida de basura insuficientes, que generan grandes cantidades de criaderos en el medio ambiente (plásticos y neumáticos inservibles entre los más importantes) [38].

En este artículo se investigó las propiedades de un modelo de crecimiento centrado en la población de hembras Aedes aegypti, incorporando una capacidad de carga logísticamente creciente para la fase acuática, vinculada a tres contenedores artificiales efectivos recurrentes, con el microclima propicio para el desarrollo de los estadios juveniles de culicidos. Los estudios entomológicos que formaron parte de nuestros antecedentes, concuerdan que la mayor magnitud y frecuencia de la infestación sucede en los baldes, los tanques y las llantas.

En el Sección 2 se presentó un sistema semiacoplado, el cual se desacopló al probar que el término acoplante, dependiente del volumen de los criaderos está acotado, debido a la capacidad de carga de los criaderos en el medio. El análisis cualitativo reveló la existencia de dos soluciones de equilibrio: el equilibrio trivial $P_{0}=(0,0)$ (pérdida de población) y el equilibrio de coexistencia $P_{1}=(3438118.293,7816495.456)$ (permanencia de generaciones de mosquitos), cuya estabilidad local y global se analizó con base en el umbral de crecimiento de $A$. aegypti: si $\mathrm{R}_{m} \leq 1$, las generaciones de mosquitos se extinguirían y si $\mathrm{R}_{m}>1$ la población perdura en el ambiente, en forma tal que a largo plazo, esta tenderá al equilibrio de coexistencia. Con la invasión de la especie, la abundancia de ninguna categoría de criadero aumenta de manera indefinida, pero en el caso más crítico podría llegar a saturar el medio ambiente.

Los resultados analíticos demostraron que si las autoridades sanitarias abordan un manejo integrado de vectores [30] en las comunidades afectadas cuyos objetivos a alcanzar sean la reducción de las capacidades de carga ambientales

a neumáticos, tanques y baldes infestados o vulnerables a las inclemencias del 
tiempo, la reducción de la productividad pupal en los criaderos epidemiológicamente importantes, la reducción de las tasas de oviposición y maduración y un incremento de las tasas de mortalidad, lograrán inhibir la propagación de los mosquitos e, indirectamente, prevenir y reducir la transmisión de enfermedades infecciosas como el dengue, el zika, el chikunguya y la fiebre amarilla en zonas endémicas.

\section{Referencias}

[1] Alcalá, L.; Quintero, J.; González-Uribe, C.; Brochero, H. (2015) "Productividad de Aedes aegypti (L.)(Diptera: Culicidae) en viviendas y espacios públicos en una ciudad endémica para dengue en Colombia", Biomédica 35(2): 258-268.

[2] Barrera, R. (2016) "Recomendaciones para la vigilancia de Aedes aegypti", Biomédica 36(3): 454-462.

[3] Barrera-Pérez, M.; Pavía-Ruiz, M.; Mendoza-Mézquita, J.; Torres Arcila, N.; Hernández-Hernández, R.; Castro-Gamboa, F.; Guedeb-Moreno, E.; Cohuo-Rodríguez, A.; Medina-Barreiro, A.; Coyoc-Cardeña, E.; GómezDantés, H.; Kroeger, A.; Vázquez-Prokopec, G.; Manrique-Saide, P. (2015) "Control de criaderos de Aedes aegypti con el programa recicla por tu bienestar en Mérida, México”, Salud Pública de México 57(3): 201-210.

[4] Bonnefoy, X.; Kampen, H.; Sweeney, K. (2008) Public Health Significance of Urban Pests. World Health Organization, Denmark.

[5] Chang, Y.; Lugo, J.; Barceló, G.; Martínez, J.; Chao, M.I. (2013) "Depósitos observados con larvas de Aedes aegypti durante el uso operacional del biolarvicida Bactivec", Revista Cubana de Medicina Tropical 65(1): 9098.

[6] Chen, S.C.; Hsieh, M.H. (2012) "Modeling the transmission dynamics of dengue fever: Implications of temperature effects", Science of the Total Environment 431(2012): 385-391.

[7] Chitnis, N.; Hyman, J.M.; Cushing, J.M. (2008) "Determining important parameters in the spread of malaria through the sensitivity analysis of a mathematical model", Bulletin of mathematical biology 70(5): 1272-1296. 
[8] Coleman, B.D.; Hsieh, Y.H.; Knowles, G.P. (1979) "On the optimal choice of $\mathrm{r}$ for a population in a periodic environment", Mathematical Biosciences 46(1-2): 71-85.

[9] Dresch, J.M.; Liu, X.; Arnosti, D.N.; Ay, A. (2010) “Thermodynamic modeling of transcription: Sensitivity analysis differentiates biological mechanism from mathematical model-induced effects", BMC Systems Biology 4(142): 1-11.

[10] Ebert, D.; Weisser, W.W. (1997) "Optimal killing for obligate killers: the evolution of life histories and Virulence of Semelparous parasites", Proceedings: Biological Sciences 264(1384): 985-991.

[11] Eiman, M.; Introini, M.V.; Ripoll, C. (2010) "Directrices para la prevención y control de Aedes aegypti", en: http:// www.msal.gob.ar/images/stories/bes/graficos/ $0000000235 \mathrm{cnt}-01$-directrices-dengue-2016.pdf, consultado el 17-Aug-2016, 9:15 a.m.

[12] Gordillo, F. (2009) "Estabilidad de sistemas no lineales basada en la teoría de Liapunov", Revista Iberoamericana de Automática e Informática 6(2): $5-16$.

[13] Gürtler, R.E.; Vezzani, D.; Carbajo, A.E.; Cardinal, M.V., Garelli, F.M.; Gaspe, M.S. (2009) "El dengue, el mosquito Aedes aegypti y la prevención”, Boletín Biológica 10: 10-16.

[14] IDEAM. (2017) "Boletin climatologico mensual", en: http: / /www.ideam.gov.co/web/tiempo-y-clima/ climatologico-mensual, consultado 10-Sep-2017, 11:00 a.m.

[15] Ikeda, S.; Yokoi, T. (1980) "Fish population dynamics under nutrient enrichment. A case of the East Seto Inland Sea", Ecological Modelling 10(34): $141-165$.

[16] Lana, R.M.; Carneiro, T.G.; Honório, N.A.; Codeço, C.T. (2014) “Seasonal and nonseasonal dynamics of Aedes aegypti in Rio de Janeiro, Brazil: Fitting mathematical models to trap data", Acta Tropica 129: 25-32. 
[17] Manrique-Saide, P.; Che-Mendoza, A.; Rizzo, N.; Arana, B.; Pilger, D.; Lenhart, A.; Kroeger, A. (2011) "Operational guide for assessing the productivity of Aedes aegypti breeding sites", en: http://www. who.int/tdr/publications/documents/ sop-pupal-surveys.pdf?ua=1, consultado el 28-Aug-2017, 4:00 p.m.

[18] Marín, C.V.; Muñoz-Loaiza, A.; Toro-Zapata, H.D; Restrepo, L.D. (2011) "Modelado de estrategias para el control químico y biológico del Aedes aegypti (Diptera: Culicidae)", Matemáticas: Enseñanza Universitaria 19(1): 63-78.

[19] Marín, R.; Díaz, M.; Álvarez, Y.; Calderón, O. (2014) "Sitios de cría de Aedes aegypti (Linnaeus) y distribución geográfica de Aedes albopictus (Skuse) en la Provincia de Limón, Costa Rica. 2012”, Revista Cubana de Medicina Tropical 66(2): 219-227.

[20] Marquetti-Fernández, M.C.; Fuster-Callaba, C.; Martín-Díaz, I. (2014) "Distribución espacial y temporal de los sitios de cría de Aedes albopictus y Aedes aegypti (Diptera: Culicidae) en Pinar del Río, Cuba", Revista Biomédica 25(2): 54-67.

[21] Meyer, P. (1994) "Bi-logistic growth", Technological Forecasting and Social Change 47: 89-102.

[22] Meyer, P.S.; Ausubel, J.H. (1999) "Carrying capacity: A model with logistically varying limits", Technological Forecasting and Social Change 61(3): 209-214.

[23] Ministerio de Salud y Protección Social de Colombia (2013) "Gestión para la vigilancia entomológica y control de la transmisión de dengue", en: http://www.ins.gov.co/temas-de-interes/dengue/ $03 \% 20$ vigilancia 20 ent omo 20 dengue.pdf, consultado el 20Aug-2016, 10:00 a.m., 8:20 p.m.

[24] Mondelo, R.E.; Tejerina, E.F.; Gauto, N.J.; Contreras, N. (2014) "Uso de membrana de drenaje para evitar acumulación de agua y posibles criaderos de Aedes aegypti (Linnaeus 1762) (Diptera: Culicidae) en neumáticos desechables", Revista Cubana de Medicina Tropical 66(2): 210-218.

[25] Murray, J.D. (2002) Mathematical Biology I: An Introduction. Springer, New York. 
[26] OMS (2009) "Estrategias de lucha antivectorial", en: http://www. who.int/denguecontrol/control_strategies/es/, consultado el 17-Aug-2015, 7:30 p.m.

[27] OMS (2016) "Dengue y dengue grave", en: http://www.who.int/ mediacentre/factsheets/fs117/es/, consultado el 15-Aug2016, 10:00 a.m.

[28] OMS (2016) "El mosquito", en: http://www.who.int/ denguecontrol/mosquito/es/, consultado el 15-Aug-2016, 10:00 a.m.

[29] OMS (2016) "Enfermedades transmitidas por vectores", en: http:// www.who.int/denguecontrol/mosquito/es/, consultado el 15-Agosto-2016, 10:00 a.m.

[30] OMS, TDR. (2009) Dengue: Guías para el Diagnóstico, Tratamiento, Prevención y Control. OMS, Bolivia.

[31] Organización Panamericana de la Salud. (2000) Los Desastres Naturales y la Protección de la Salud. OPS, Washington, D.C.

[32] Perko, L. (2013) Differential Equations and Dynamical Systems, 3rd Edition. Springer Science \& Business Media, Berlín.

[33] Rey, J.; Lounibos, P. (2015) "Ecología de Aedes aegypti y Aedes albopictus en América y transmisión de enfermedades", Biomédica 35(2): 177-185.

[34] Rogovchenko, S.P.; Rogovchenko, Y.V. (2009) "Effect of periodic environmental fluctuations on the Pearl-Verhulst model", Chaos, Solitons \& Fractals 39(3):1169-1181.

[35] Safuan, H.; Towers, I.N.; Jovanoski, Z.; Sidhu, H.S. (2011) "A Simple model for the total microbial biomass under occlusion of healthy human skin”, in: MODSIM2011 (Ed.) 19th International Congress on Modelling and Simulation, Modelling and Simulation Society of Australia and New Zealand, Australia: 733-739.

[36] Stein, M.; Oria, G.I.; Almirón, W.R. (2002) "Principales criaderos para Aedes aegypti y culícidos asociados, Argentina", Revista de Saúde Pública 36(5): 627-630. 
[37] Unicef (2009) "Participación social en la prevención del dengue. Guía para el promotor", en: https://www.unicef.org/argentina/ spanish/MANUAL_DENGUE_A5-FINAL_corregido.pdf, consultado el 18-Aug-2016, 10:30 p.m.

[38] WHO (2017) "Global vector control response", en: http:// www.who.int/malaria/areas/vector_control/ global-vector-control-response/en/, consultado el 11May-2017, 4:05 p.m.

[39] Wiggins, S. (2003) Introduction to Applied Nonlinear Dynamical Systems and Chaos. Springer-Verlag, New York.

[40] Yang, H.M.; da Graça, M.L.; Galvani, K.C.; Macoris, M.T. (2011) "Follow up estimation of Aedes aegypti entomological parameters and mathematical modellings", Biosystems 103(3): 360-371.

[41] Yang, H.M.; Ferreira, C.P. (2008) "Assessing the effects of vector control on dengue transmission", Applied Mathematics and Computation 198(1): 401-413.

[42] Zapata, L.; Mari, E.; Stein, M.; Gorodner, J. (2002) “Criaderos de Aedes aegypti y la culicidofauna asociada (Diptera: Culicidae) en la Ciudad de Corrientes", Revista Facultad de Medicina UNNE, 31 (4): 370-77.

[43] Zapata-Peniche, A.; Manrique-Saide, P.; Rebollar-Téllez, E.A.; CheMendoza, A.; Dzul-Manzanilla, F. (2007) "Identificación de larvas de mosquitos (Diptera: Culicidae) de Mérida, Yucatán, México y sus principales criaderos", Revista Biomed 18(1): 3-17. 
